\title{
Modelling the Inclusion of Swelling Pressure in a Tissue Level Poroviscoelastic Model of Cartilage Deformation
}

\author{
JONATHAN P WHITELEY \\ Department of Computer Science, University of Oxford, \\ Wolfson Building, Parks Road, Oxford OX1 3QD, UK. \\ EAMONN A GAFFNEY \\ Mathematical Institute, University of Oxford, \\ Andrew Wiles Building, Radcliffe Observatory Quarter, Woodstock Rd, Oxford OX2 6GG, UK.
}

[Received on]

\begin{abstract}
Swelling pressure in the interstitial fluid within the pores of cartilage tissue is known to have a significant effect on the rheology of cartilage tissue. The swelling pressure varies rapidly within thin regions inside pores known as Debye layers, caused by the presence of fixed charge, as observed in cartilage. Tissue level calculation of cartilage deformation therefore requires resolution of three distinct spatial scales: the Debye lengthscale within individual pores; the lengthscale of an individual pore; and the tissue lengthscale. We use asymptotics to construct a leading order approximation to the swelling pressure within pores, allowing the swelling pressure to be systematically included within a fluid-solid interaction model at the level of pores in cartilage. We then use homogenisation to derive tissue level equations for cartilage deformation that are very similar to those governing the finite deformation of a poroviscoelastic body. The equations derived permit the spatial variations in porosity and electric charge that occur in cartilage tissue. Example solutions are then used to confirm the plausibility of the model derived and to consider the impact of fixed charge heterogeneity, illustrating that local fixed charge loss is predicted to increase deformation gradients under confined compression away from, rather than at, the site of loss.
\end{abstract}

Keywords: Poisson-Nernst-Planck equations, cartilage, poroviscoelasticity, homogenisation

\section{Introduction}

Cartilage is often modelled as a poroviscoelastic (or poroelastic) multiphase material, where the solid scaffold comprises charged proteoglycans, mainly aggrecans; see, for example, [27] and references therein. The pores in this material are filled with interstitial fluid containing free ions, predominantly but not exclusively sodium and chloride ions, with concentrations that depend on the charge on the surface of the solid scaffold. These free ions result in a swelling pressure that is sufficiently large that it fundamentally affects cartilage mechanics and must be included in tissue level models of cartilage deformation. There are two main challenges when integrating the swelling pressure into a tissue level model of cartilage tissue. The first of these is modelling the thin electrical films within the cartilage pores, known as Debye layers, sufficiently accurately to allow a spatially accurate description of the swelling pressure within the pores of the cartilage. The second challenge is to incorporate the swelling pressure into a tissue scale poroelastic or poroviscoelastic model, allowing predictions of the deformation and stresses arising in cartilage tissue mechanics.

Swelling pressure is usually incorporated into tissue level models of cartilage using Donnan theory $[25,30,31]$ or the Poisson-Boltzmann equation [8]. Both approaches acknowledge that the concentration of both sodium and chloride ions vary significantly at the lengthscale of an individual pore. Donnan

(c) The author 2008. Published by Oxford University Press on behalf of the Institute of Mathematics and its Applications. All rights reserved. 
theory assumes that the fluid within the pores may be divided into two regions - a thin Debye layer in the vicinity of the charged solid scaffold where ion concentrations result in a non-zero net charge, and an outer charge-neutral region in the remainder of the fluid. The free ion concentration within the Debye layer is calculated by taking account of both Donnan equilibrium at the interface between these two regions within the fluid, and the charge on the scaffold. The resulting ion concentrations in the Debye layer are then used to calculate the swelling pressure. The Poisson-Boltzmann equation offers a more detailed and physically realistic description of the free ion concentraions, representing these concentrations by continuous functions and taking account of the electrical potential caused by these ions when balancing the charge on the scaffold.

However, in going to higher lengthscales, there has been some debate on how swelling pressure, which we have noted varies significantly within pores of the scaffold, should be included in a tissue level model of cartilage tissue. Some studies model the swelling pressure as being an extra pressure that is only present in the fluid phase [25, 27]; other studies model the swelling pressure as a potential whose gradient is an external force that is applied to the whole body [28, 30, 35]. A further complication is that the deformations are sufficiently large that finite deformation viscoelasticity or elasticity (see, for example, [24]) must be used rather than linear viscoelasticity or elasticity [34]. After deciding how to include swelling pressure in the model, a stress tensor for the composite body is required. Most multiphase models of biological tissue that comprise a solid phase and a fluid phase assume that the tissue stress tensor is given by a weighted average of the contribution from the solid and fluid phases $[4,5,10,33,34]$. We also need to specify constitutive relationships to allow the stress tensor to be calculated. The solid scaffold is usually modelled as an elastic or viscoelastic body. If the cartilage scaffold is modelled as being viscoelastic then the Voigt formulation, where the scaffold acts as a fluid on very short timescales but as an elastic solid on longer timescales, is used rather than the Maxwell formulation.

We have noted that the concentrations of free sodium and chloride ions vary significantly within thin Debye layers in the pores of the solid cartilage scaffold. Furthermore, the pore size within the cartilage is much smaller than the tissue level lengthscale of cartilage. An accurate representation of swelling pressure within a tissue level model of cartilage deformation therefore requires us to resolve three clearly separated lengthscales - the Debye layer thickness, the pore size, and the tissue lengthscale. In this study our aim is to use asymptotics to derive equations that take account of all three of these spatial scales, so as to examine the predictions of homogenisation theory for how swelling pressure and fixed charge, and especially their gradients as well as gradients of other material properties, manifest in terms of tissue level equations and predicted behaviour.

To proceed, we need to take account of many entities at different lengthscales and thus, for clarity, we begin by defining the notation used throughout this manuscript in Section 2. Having defined our notation we then write down a microscale model of the fluid flow and electrochemistry in the interstitial fluid in the cartilage pores in Section 3. A detailed description of the microstructure of articular tissue is given in [35]. Proteoglycan monomers consist of a central core that many chondroitin sulfate chains branch from. Networks of proteoglycans are formed when many proteoglycan monomers bind to a filament chain of hyaluronate. These networks of proteoglycans then interact with networks of collagen to form a composite cartilage scaffold (see Figure 1). As a first approximation we will model this scaffold as a viscoelastic solid with a negatively charged surface, with the latter representing the negative charges of the glycoaminoglycans within the chondroitin sulphate arms. This corresponds to the simplest surface charge - model of Dean et al. [13] and Han et al. [20], though with the surface charge density displaced to the envelope formed by the ends of the glycoaminoglycans (see Figure 1), which improves the match of predicted electrical forces between the surface charge models and a more complex - 
(a)

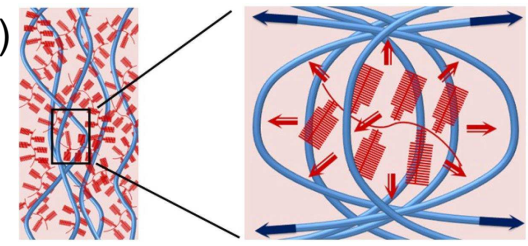

(c)

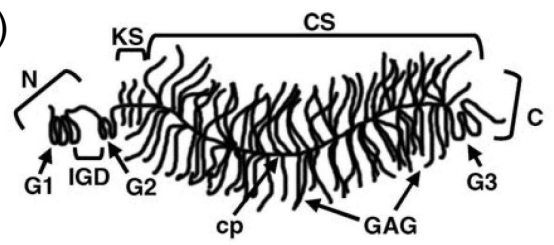

(b)

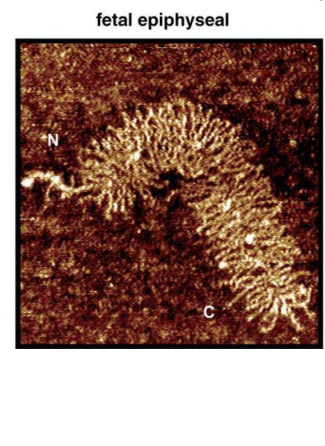

\section{mature nasal}

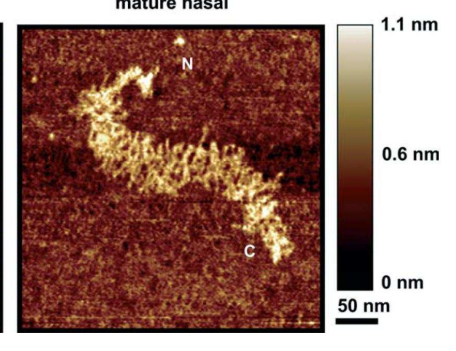

FIG. 1. The structure of cartilage. (a) A schematic of cartilage molecular structure with collagen in blue, with hyaluronic acid backbones, for instance the red-curve depicted in the blow-up, which are decorated with aggregan. (b) Atomic force microscopy images of fetal epiphyseal and nasal mucosal aggrecans, with the $\mathrm{N}$ (amine) and $\mathrm{C}$ (carboxyl) termini labelled. (c) The detailed molecular structure of aggrecans, which binds to hyaluronic acid via the N-terminus from which emerges the G1 and G2 globular domains, followed by core protein (cp) dressed by keratin sulphate (KS) and chondroitin sulphate (CS) glycoaminogkycan (GAG) chains, and ending with the G3 globular domain and C terminus. Figure (a) is reproduced from [27], and Figures (b) and (c) are from [37], permissions pending.

We proceed to exploit the observation that the length of the glycoaminoglycan chains, at roughly $30-40 \mathrm{~nm}$ as seen in Figure 1, is much larger than the Debye layer thickness of roughly $1 \mathrm{~nm}$ at physiologial ion concentrations [42]. This ratio of lengthscales introduces a small parameter, allowing the construction of a leading order approximation to the fluid flow and forces exerted on the interface with the solid, taking account of the swelling pressure in interstitial fluid that arises due to dissolved ions. Rather than assume that the tissue stress tensor is given by a weighted average of the contribution from the solid and fluid phases, we use the leading order approximation to the swelling pressure to write down a coupled fluid-structure interaction problem at the microscale in Section 4 . We then use homogenisation to derive the macroscale poroviscoelasticity equations that take account of swelling pressure. Our approach is similar in spirit to that of other authors who have used homogenisation to derive general macroscale equations governing a poroelastic body [7, 38, 41]. There are, however, three differences between these works and the model presented here. First, we introduce the leading order swelling pressure into our derivation of the macroscale equations. Second, we assume that the solid scaffold in cartilage tissue is completely incompressible as is suggested by experiments [2]; any change in the volume of the composite material is then entirely due to fluid being squeezed in or out. Finally, we allow the scaffold to be viscoelastic; the resulting macroscale equations then model cartilage as a poroviscoelastic body, although the viscoelastic scaffold can easily be replaced by an elastic scaffold, resulting in macroscale equations for a poroelastic body. We then summarise the macroscale equations in Section 5, presenting illustrative solutions and comparisons with other work, before summarising our conclusions in Section 6. 


\section{Notation used}

In this study we will require dimensional and dimensionless variables, and both Eulerian and Lagrangian coordinates. The use of homogenisation will require both a microscale coordinate system and a macroscale coordinate system. Furthermore we will also need a coordinate system that represents the Debye layer within the microscale coordinate system. To aid the clarity of this manuscript we begin by explaining the notation used. We will use upper case letters to denote Lagrangian coordinates, and the corresponding lower case letter to denote Eulerian coordinates. Indices that refer to the Lagrangian frame will be upper case letters, and indices referring to the Eulerian frame will be lower case letters. We use superscript asterisks to denote dimensional quantities; quantities without an asterisk are the corresponding dimensionless quantity. At the microscale we model the material as comprising, in Eulerian coordinates, an interconnected solid phase occupying the region $\Omega_{s}^{*}$, and an interconnected fluid phase occupying the region $\Omega_{f}^{*}$. The boundary between these regions is denoted by $\partial \Omega_{f s}^{*}$. The fluid phase is assumed to be an incompressible Newtonian fluid containing both sodium and chloride ions in solution. In Lagrangian coordinates these regions are denoted by $\Omega_{s, 0}^{*}, \Omega_{f, 0}^{*}$ and $\partial \Omega_{f s, 0}^{*}$ respectively. We denote the dimensional microscale Eulerian coordinate by $\mathbf{y}^{*}$, and the dimensional macroscale Eulerian coordinate by $\mathbf{x}^{*}$. The corresponding Lagrangian coordinates are $\mathbf{Y}^{*}$ and $\mathbf{X}^{*}$. When using the gradient operator we will use a subscript to identify the coordinates it is with respect to, i.e. $\nabla_{\mathbf{y}^{*}}$ is the gradient operator with respect to the microscale coordinate system $\mathbf{y}^{*}$. We will see later that, in the microscale coordinate system, we require a scaled dimensionless coordinate that represents the distance into the Debye layer in the direction of the vector normal to the interface between the solid and the fluid; this coordinate will be denoted by $\xi$.

We will frequently require use of a vector that is normal to the interface between the solid and fluid regions. The vector $\mathbf{n}$ is defined to be the vector in Eulerian coordinates that is normal to the interface, of unit length, and points from the solid region into the fluid region. The corresponding vector in Lagrangian coordinates is denoted by $\mathbf{N}$.

The notation presented in this section, and the three distinct scales modelled, are illustrated in Figure 2. We will use the Einstein summation convention throughout this manuscript unless otherwise stated.

\section{Modelling the Debye layers}

In this section we derive a model of the swelling pressure that is caused by charged ions within the interstitial fluid. We first write down the dimensional governing equations. These equations comprise the Navier-Stokes equations [1] that describe fluid flow, and the Poisson-Nernst-Planck equations [36] that govern electrochemistry. We then nondimensionalise these equations and identify the existence of Debye layers, i.e. boundary layers in the ion concentration in the vicinity of the interface between the fluid and solid. We then follow [43] and derive a leading order approximation to the solution of the governing equations. These leading order approximations will then be used to calculate both the leading order stress tensor in the fluid and the leading order contribution to the Lorentz force induced by the charged interface, which will be used when deriving our tissue level poroviscoelastic model. Throughout this section we will use Eulerian coordinates. 


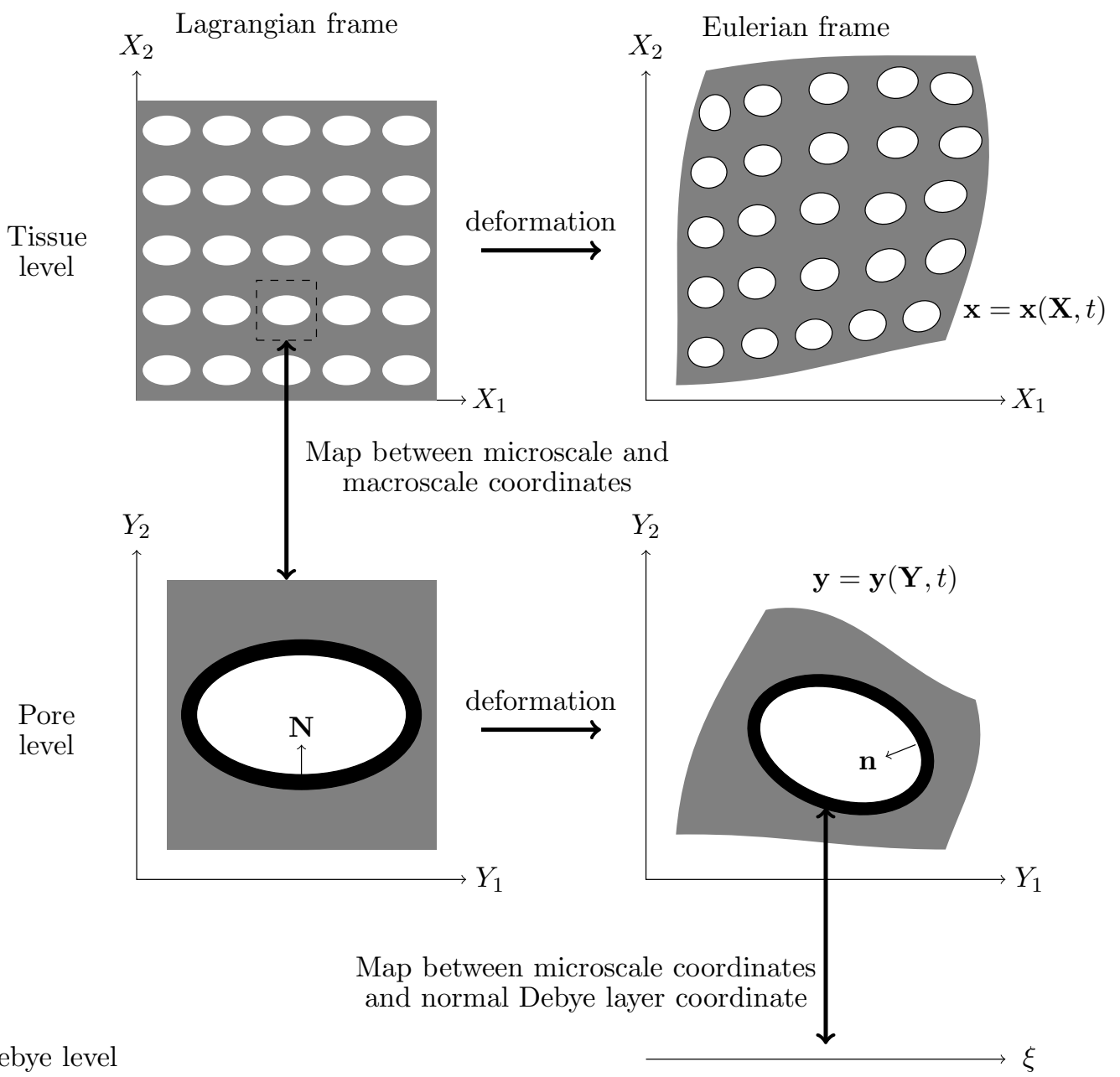

FIG. 2. A schematic of the three scales considered in this paper. At the tissue scale, grey regions represent the solid, and white regions represent the fluid. At the pore scale, grey regions represent the solid, black regions represent the Debye layer within the fluid, and white regions represent the remainder of the fluid. See Section 2 for definition of the notation used. For clarity we do not distinguish between dimensional and dimensionless quantities in this figure.

\subsection{The dimensional equations}

The governing equations in the fluid are given by, for $\mathbf{y}^{*} \in \Omega_{f}^{*}$ :

$$
\begin{aligned}
\rho_{f}^{*}\left(\frac{\partial \mathbf{v}^{*}}{\partial t^{*}}+\left(\mathbf{v}^{*} \cdot \nabla_{\mathbf{y}^{*}}\right) \mathbf{v}^{*}\right) & =-\nabla_{\mathbf{y}^{*}}\left(p^{*}+p_{\mathrm{swell}}^{*}\right)+\mu_{f}^{*} \nabla_{\mathbf{y}^{*}}^{2} \mathbf{v}^{*}, \\
\nabla_{\mathbf{y}^{*}} \cdot \mathbf{v}^{*} & =0,
\end{aligned}
$$


where $\rho_{f}^{*}$ is the constant density of the fluid, $t^{*}$ is time, $\mu_{f}^{*}$ is the dynamic viscosity of the fluid, $\mathbf{v}^{*}$ is the fluid velocity, $p^{*}$ is hydrodynamic pressure that is also a Lagrange multiplier used to enforce the incompressibility constraint given by Eq. (3.2), and $p_{\text {swell }}^{*}$ is the swelling pressure caused by dissolved sodium and chloride ions. The gradient of the swelling pressure is equal to the Lorentz force and so, as sodium ions are positive ions with a valence of 1 and chloride ions are negative ions with a valence of 1 ,

$$
\nabla_{\mathbf{y}^{*}} p_{\mathrm{swell}}^{*}=-F^{*}\left(N^{*}-C^{*}\right) \nabla_{\mathbf{y}^{*}} \phi^{*}
$$

where $N^{*}$ is the concentration of sodium ions, $C^{*}$ is the concentration of chloride ions, $\phi^{*}$ is the electrical potential in the fluid, and $F^{*}$ is Faraday's constant. The ion concentrations and potential satisfy the Poisson-Nernst-Planck equations given by, for $\mathbf{y}^{*} \in \Omega_{f}^{*}$ :

$$
\begin{aligned}
\frac{\partial N^{*}}{\partial t^{*}}+\nabla_{\mathbf{y}^{*}} \cdot\left(N^{*} \mathbf{v}^{*}-D_{N}^{*}\left(\nabla_{\mathbf{y}^{*}} N^{*}+\frac{F^{*} N^{*}}{R^{*} T^{*}} \nabla_{\mathbf{y}^{*}} \phi^{*}\right)\right) & =0 \\
\frac{\partial C^{*}}{\partial t^{*}}+\nabla_{\mathbf{y}^{*}} \cdot\left(C^{*} \mathbf{v}^{*}-D_{C}^{*}\left(\nabla_{\mathbf{y}^{*}} C^{*}-\frac{F^{*} C^{*}}{R^{*} T^{*}} \nabla_{\mathbf{y}^{*}} \phi^{*}\right)\right) & =0, \\
-\nabla_{\mathbf{y}^{*}} \cdot\left(\varepsilon_{f}^{*} \nabla_{\mathbf{y}^{*}} \phi^{*}\right) & =F^{*}\left(N^{*}-C^{*}\right),
\end{aligned}
$$

where $D_{N}^{*}, D_{C}^{*}$ are the diffusion coefficients for sodium and chloride ions in the fluid phase, $R^{*}$ is the universal gas constant, $T^{*}$ is absolute temperature, and $\varepsilon_{f}^{*}$ is the permittivity of the interstitial fluid. Remembering that in Section 2 we defined $\mathbf{n}$ to be the unit normal vector pointing out of the solid into the fluid, i.e. the inward pointing normal vector within the fluid, boundary conditions are, for $\mathbf{y}^{*} \in \partial \Omega_{f s}^{*}$ :

$$
\begin{aligned}
\left(N^{*} \mathbf{v}^{*}-D_{N}^{*}\left(\nabla_{\mathbf{y}^{*}} N^{*}+\frac{F^{*} N^{*}}{R^{*} T^{*}} \nabla_{\mathbf{y}^{*}} \phi^{*}\right)\right) \cdot \mathbf{n} & =0 \\
\left(C^{*} \mathbf{v}^{*}-D_{C}^{*}\left(\nabla_{\mathbf{y}^{*}} C^{*}-\frac{F^{*} C^{*}}{R^{*} T^{*}} \nabla_{\mathbf{y}^{*}} \phi^{*}\right)\right) \cdot \mathbf{n} & =0, \\
-\varepsilon_{f}^{*} \nabla_{\mathbf{y}^{*}} \phi^{*} \cdot \mathbf{n} & =Q^{*}
\end{aligned}
$$

where $\mathbf{v}_{f s}^{*}$ is the velocity of the interface, and $Q^{*}$ is the surface charge density on the solid due to the fixed charge proteoglycan aggregates. We remark that $Q^{*}<0$ for cartilage. We also note that the dimensional Cauchy stress tensor for the fluid, $\sigma^{*}$, has entries given by

$$
\sigma_{i j}^{*}=-\left(p^{*}+p_{\text {swell }}^{*}\right) \delta_{i j}+\frac{\mu_{f}^{*}}{2}\left(\frac{\partial v_{i}^{*}}{\partial x_{j}^{*}}+\frac{\partial v_{i}^{*}}{\partial x_{j}^{*}}\right), \quad i, j=1,2,3 .
$$

\subsection{The dimensionless equations}

We use the nondimensionalisation given by

$$
\begin{aligned}
& t^{*}=\tau^{*} t, \quad \mathbf{y}^{*}=d^{*} \mathbf{y}, \quad \mathbf{v}^{*}=V^{*} \mathbf{v}, \quad p^{*}, p_{\text {swell }}^{*}=\frac{\mu_{f}^{*} V^{*}}{d^{*}} p, p_{\text {swell }}, \quad D_{n, c}^{*}=\bar{D}^{*} D_{n, c}, \\
& N^{*}=\Pi_{0}^{*} N, \quad C^{*}=\Pi_{0}^{*} N, \quad \phi^{*}=\frac{R^{*} T^{*}}{F^{*}} \phi, \quad Q^{*}=\frac{R^{*} T^{*} \varepsilon_{f}^{*}}{F^{*} d^{*} \delta} Q,
\end{aligned}
$$


where $\tau^{*}$ is a representative timescale, $d^{*}$ is a typical pore spacing, $V^{*}$ is a representative velocity, $\Pi_{0}^{*}$ is a typical value of the concentration of sodium and chloride ions, and $\bar{D}^{*}$ is a typical value of the diffusion coefficients for sodium and chloride ions. Setting

$$
\begin{aligned}
\tau^{*} & =\frac{d^{*^{2}}}{\bar{D}^{*}}, \\
V^{*} & =\frac{d^{*}}{\tau^{*}}=\frac{\bar{D}^{*}}{d^{*}},
\end{aligned}
$$

and defining the dimensionless parameter $\delta$ by

$$
\delta^{2}=\frac{\varepsilon_{f}^{*} R^{*} T^{*}}{d^{*^{2}} F^{*^{2}} \Pi_{0}^{*}}
$$

Eqs. (3.1)-(3.6) become, for $\mathbf{y} \in \Omega_{f}$ :

$$
\begin{aligned}
\operatorname{Re}_{l}\left(\frac{\partial \mathbf{v}}{\partial t}+\left(\mathbf{v} \cdot \nabla_{\mathbf{y}}\right) \mathbf{v}\right) & =-\nabla_{\mathbf{y}}\left(p+p_{\text {swell }}\right)+\nabla_{\mathbf{y}}^{2} \mathbf{v} \\
\nabla_{\mathbf{y}} \cdot \mathbf{v} & =0, \\
\nabla_{\mathbf{y}} p_{\text {swell }} & =-\mathscr{A}(N-C) \nabla_{\mathbf{y}} \phi \\
\frac{\partial N}{\partial t}+\nabla_{\mathbf{y}} \cdot\left(N \mathbf{v}-D_{N}\left(\nabla_{\mathbf{y}} N+N \nabla_{\mathbf{y}} \phi\right)\right) & =0, \\
\frac{\partial C}{\partial t}+\nabla_{\mathbf{y}} \cdot\left(C \mathbf{v}-D_{C}\left(\nabla_{\mathbf{y}} C-C \nabla_{\mathbf{y}} \phi\right)\right) & =0, \\
-\nabla_{\mathbf{y}}^{2} \phi & =\frac{1}{\delta^{2}}(N-C),
\end{aligned}
$$

where the dimensionless parameters $\mathscr{A}$ and $\operatorname{Re}_{l}$ (the local Reynolds number), are given by

$$
\mathscr{A}=\frac{\Pi_{0}^{*} R^{*} T^{*} d^{*^{2}}}{\mu_{f}^{*} \bar{D}^{*}}, \quad \operatorname{Re}_{l}=\rho_{f}^{*} V^{*} d^{*} / \mu_{f}^{*}=\rho_{f}^{*} \bar{D}^{*} / \mu_{f}^{*}
$$

The boundary conditions, Eqs. (3.7)-(3.10), become, for $\mathbf{y} \in \partial \Omega_{f s}$ :

$$
\begin{aligned}
\mathbf{v} & =\mathbf{v}_{f s}, \\
\left(N \mathbf{v}-D_{N}\left(\nabla_{\mathbf{y}} N+N \nabla_{\mathbf{y}} \phi\right)\right) \cdot \mathbf{n} & =0, \\
\left(C \mathbf{v}-D_{C}\left(\nabla_{\mathbf{y}} C-C \nabla_{\mathbf{y}} \phi\right)\right) \cdot \mathbf{n} & =0, \\
-\nabla_{\mathbf{y}} \phi \cdot \mathbf{n} & =\frac{1}{\delta} Q .
\end{aligned}
$$

Representative parameter values are given in Table 1. We note that interstitial fluid is reported to be substantially less viscous than synovial fluid [32]; thus, noting synovial fluid viscosity at the shear rates supported by cartilage is about $0.1 \mathrm{~kg} \mathrm{~m}^{-1} \mathrm{~s}^{-1}[3,26]$, this gives an upper bound for the interstitial fluid viscosity, $\mu_{f}^{*}$. A lower viscosity bound is that of water, which is very roughly $0.001 \mathrm{~kg} \mathrm{~m}^{-1} \mathrm{~s}^{-1}$, and we take an intermediate value, $\mu_{f}^{*} \approx 0.01 \mathrm{~kg} \mathrm{~m}^{-1} \mathrm{~s}^{-1}$. Combined with the other parameter estimates of Table (1), the dimensionless parameters appearing in Eqs. (3.13)-(3.21) are approximately $\delta \approx 0.129$, $\mathscr{A} \approx 2.63$, and $\operatorname{Re}_{l} \approx 10^{-5}$. 


\begin{tabular}{lll}
\hline & & \\
Parameter & Value & Reference \\
\hline & & \\
$d^{*}$ & $6 \times 10^{-9} \mathrm{~m}$ & {$[37]$} \\
$F^{*}$ & $9.65 \times 10^{4} \mathrm{C} \mathrm{mol}^{-1}$ & {$[43]$} \\
$R^{*}$ & $8.31 \mathrm{C} \mathrm{V} \mathrm{mol}^{-1} \mathrm{~K}^{-1}$ & {$[43]$} \\
$T^{*}$ & $293 \mathrm{~K}$ & {$[8]$} \\
$\varepsilon_{f}^{*}$ & $78.3 \varepsilon_{0}^{*}$ & {$[8]$} \\
$\varepsilon_{0}^{*}$ & $8.85 \times 10^{-12} \mathrm{~F} \mathrm{~m}^{-1}$ & {$[8]$} \\
$\Pi_{0}^{*}$ & $3 \times 10^{2} \mathrm{~mol} \mathrm{~m}^{-3}$ & {$[28]$} \\
$\rho_{f}^{*}$ & $10^{3} \mathrm{~kg} \mathrm{~m}^{-3}$ & {$[29]$} \\
$\mu_{f}^{*}$ & $10^{-2} \mathrm{~kg} \mathrm{~m}^{-1} \mathrm{~s}^{-1}$ & See text and $[3,26]$ \\
$\bar{D}^{*}$ & $10^{-9} \mathrm{~m}^{2} \mathrm{~s}^{-1}$ & {$[14]$} \\
\hline
\end{tabular}

Table 1. Parameter values for the microscale model

We have not been able to obtain an estimate for a value for $Q^{*}$, the surface charge density on the solid due to the proteoglycan aggregates. Previous work that has considered the contribution from the charge on the proteoglycans has been concerned with a much longer lengthscale than the microstructure discussed here. As a consequence the charge has been modelled as a continuum, and values are given in the units of Coulombs per unit volume, rather than Coulombs per unit surface area. We make the assumption that the dimensionless charge per unit area satisfies $Q=\mathscr{O}(1)$, as we will see that this nondimensionalisation allows the leading order solution to Eqs. (3.13)-(3.22) to exhibit the Debye layers that are believed to occur.

\subsection{Multiple scales analysis}

The equations given by Eqs. (3.13)-(3.22) contain three dimensionless parameters: $\delta, \mathscr{A}$, and $\operatorname{Re}_{l}$. The parameter $\mathscr{A}$ is $\mathscr{O}(1)$, and so clearly must be retained when calculating an approximate solution. We have two further parameters: $\delta=\mathscr{O}(0.1)$, and $\operatorname{Re}_{l}=\mathscr{O}\left(10^{-5}\right)$. On inspection of Eqs. (3.13)-(3.22) we observe the potential of a boundary layer due the small parameter $\delta$. For this reason, and given the relative sizes of $\delta$ and $\operatorname{Re}_{l}$, we first let $\operatorname{Re}_{l} \rightarrow 0$ and then construct an asymptotic solution in powers of $\delta$ and take the limit $\delta \rightarrow 0$.

Away from any boundaries we expand the outer solution as regular asymptotic expansions, using the superscript " $o$ " to denote the outer solution:

$$
\begin{aligned}
\mathbf{v}^{o} & =\mathbf{v}_{0}^{o}+\delta \mathbf{v}_{1}^{o}+\delta^{2} \mathbf{v}_{2}^{o}+\ldots \\
p^{o} & =p_{0}^{o}+\delta p_{1}^{o}+\delta^{2} p_{2}^{o}+\ldots \\
p_{\text {swell }}^{o} & =p_{\text {swell }, 0}^{o}+\delta p_{\text {swell }, 1}^{o}+\delta^{2} p_{\text {swell }, 2}^{o}+\ldots \\
N^{o} & =N_{0}^{o}+\delta N_{1}^{o}+\delta^{2} N_{2}^{o}+\ldots \\
C^{o} & =C_{0}^{o}+\delta C_{1}^{o}+\delta^{2} C_{2}^{o}+\ldots \\
\phi^{o} & =\phi_{0}^{o}+\delta \phi_{1}^{o}+\delta^{2} \phi_{2}^{o}+\ldots
\end{aligned}
$$

The leading order contributions to Eqs. (3.16)-(3.18) admit a solution that yields both electroneutrality 
and no electric fields away from the Debye layer, given by

$$
N_{0}^{o}(\mathbf{x})=C_{0}^{o}(\mathbf{x})=\bar{N}, \quad \phi_{0}^{o}(\mathbf{x})=\Phi,
$$

where $\bar{N}, \Phi$ are constants. Using Eq. (3.15) the leading order swelling pressure is given by

$$
p_{\text {swell }, 0}^{o}=\text { constant. }
$$

The constant solutions given by Eq. (3.23) do not satisfy the boundary condition given by Eq. (3.22). We have noted that the structure of Eqs. (3.13)-(3.22) suggest the existence of a Debye boundary layer of thickness $\delta$ adjacent to the interface with the solid scaffold. As discussed in Section 1 we will take it that the radius of curvature of the solid region is much greater than the width of the Debye layer. This allows us to assume that the boundary $\partial \Omega_{f s}$ is flat, avoiding the need to introduce curvilinear coordinates; we are, in essence, assuming that the effect of curvature of the solid surface affects only higher order terms in the asymptotic expansion. Orienting coordinates so that $\partial \Omega_{f s}$ is the surface $y_{1}=0$, with the fluid occupying the region $y_{1}>0$, we rescale coordinates in the Debye layer using

$$
y_{1}=\delta \xi
$$

leaving $y_{2}$ and $y_{3}$ unchanged, and write the solution in the Debye layer as asymptotic expansions, using the superscript " $D$ " to denote the Debye layer solution:

$$
\begin{aligned}
v_{i}^{D} & =v_{i, 0}^{D}+\delta v_{i, 1}^{D}+\delta^{2} v_{i, 2}^{D}+\ldots \\
p^{D} & =p_{0}^{D}+\delta p_{1}^{D}+\delta^{2} p_{2}^{D}+\ldots \\
p_{\text {swell }}^{D} & =p_{\text {swell }, 0}^{D} \delta p_{\text {swell }, 1}^{D}+\delta^{2} p_{\text {swell }, 2}^{D}+\ldots \\
N^{D} & =N_{0}^{D}+\delta N_{1}^{D}+\delta^{2} N_{2}^{D}+\ldots \\
C^{D} & =C_{0}^{D}+\delta C_{1}^{D}+\delta^{2} C_{2}^{D}+\ldots \\
\phi^{D} & =\phi_{0}^{D}+\delta \phi_{1}^{D}+\delta^{2} \phi_{2}^{D}+\ldots
\end{aligned}
$$

The $\mathscr{O}\left(\delta^{-1}\right)$ contribution to Eq. (3.14) gives

$$
\frac{\partial v_{1,0}^{D}}{\partial \xi}=0
$$

and the $\mathscr{O}\left(\delta^{-2}\right)$ contributions to the second and third components of Eq. (3.13) give

$$
\frac{\partial^{2} v_{2,0}^{D}}{\partial \xi^{2}}=0, \quad \frac{\partial^{2} v_{3,0}^{D}}{\partial \xi^{2}}=0 .
$$

We will match the Debye layer solution as $\xi \rightarrow \infty$ with the outer solution, and so the Debye layer solution must be bounded in the limit $\xi \rightarrow \infty$. Eqs. (3.25) and (3.26) then allow us to deduce that all components of the leading order velocity in the Debye layer are independent of $\xi$ and so we may write

$$
v_{1,0}^{D}=f_{1}\left(y_{2}, y_{3}\right), \quad v_{2,0}^{D}=f_{2}\left(y_{2}, y_{3}\right), \quad v_{3,0}^{D}=f_{3}\left(y_{2}, y_{3}\right) .
$$

Applying the boundary condition given by Eq. (3.19) then allows us to write

$$
\lim _{\mathbf{r} \rightarrow \partial \Omega_{f s}} \mathbf{v}_{0}^{o}=\mathbf{v}_{0}^{D}=\mathbf{v}_{f s} .
$$


We now derive an equation for the leading order pressure solution in the Debye layer. First we substitute the expressions for $v_{2,0}^{D}$ and $v_{3,0}^{D}$ given by Eq. (3.27) into the $\mathscr{O}(1)$ contribution to Eq. (3.14):

$$
\begin{aligned}
\frac{\partial v_{1,1}^{D}}{\partial \xi} & =-\frac{\partial v_{2,0}^{D}}{\partial y_{2}}-\frac{\partial v_{3,0}^{D}}{\partial y_{3}} \\
& =\frac{\partial f_{2}}{\partial y_{2}}+\frac{\partial f_{3}}{\partial y_{3}}
\end{aligned}
$$

As the right hand side of the equation immediately above is independent of $\xi$, and $v_{1,1}^{D}$ is finite, integrating over the Debye layer yields

$$
\left[\frac{\partial v_{1,1}^{D}}{\partial \xi}\right]_{0}^{\infty}=0
$$

To complete our derivation of the leading order pressure in the Debye layer we take the limit $\operatorname{Re}_{l} \rightarrow 0$ in the $\mathscr{O}\left(\delta^{-1}\right)$ contribution to Eq. (3.13):

$$
\frac{\partial^{2} v_{1,1}^{D}}{\partial \xi^{2}}-\frac{\partial}{\partial \xi}\left(p_{0}^{D}+p_{\text {swell }, 0}^{D}\right)=0 .
$$

Integrating, using Eq. (3.29), and matching with the outer solution gives

$$
p_{0}^{D}+p_{\mathrm{swell}, 0}^{D}=\lim _{\mathbf{r} \rightarrow \partial \Omega_{f s}}\left(p_{0}^{o}+p_{\mathrm{swell}, 0}^{o}\right)
$$

and we remind ourselves that $p_{\text {swell, }, 0}^{o}$ is constant, as predicted by Eq. (3.24). A useful consequence of Eqs. (3.28) and (3.30) is that they predict that both the velocity, and the sum of the hydrostatic and swelling pressures, are unaffected by the Debye layer at leading order. The Cauchy stress tensor given by Eq. (3.11) is therefore also unaffected by the Debye layer at leading order, and so the leading order stress tensor in the Debye layer may be calculated using the leading order outer solution.

3.3.1 Estimating the surface charge on the interface. We remarked earlier that we were unable to obtain an estimate for the surface charge on the interface between the fluid and ths solid. We now derive the leading order approximation to the ion concentrations and potential in the Debye layer, which will allow us to estimate the charge from quantities that we do have an estimate of. The $\mathscr{O}\left(\delta^{-2}\right)$ contributions to Eqs. (3.16)-(3.18) in the Debye layer are

$$
\begin{aligned}
\frac{\partial}{\partial \xi}\left(\frac{\partial N_{0}^{D}}{\partial \xi}+N_{0}^{D} \frac{\partial \phi_{0}^{D}}{\partial \xi}\right) & =0 \\
\frac{\partial}{\partial \xi}\left(\frac{\partial C_{0}^{D}}{\partial \xi}-C_{0}^{D} \frac{\partial \phi_{0}^{D}}{\partial \xi}\right) & =0, \\
-\frac{\partial^{2} \phi_{0}^{D}}{\partial \xi^{2}} & =N_{0}^{D}-C_{0}^{D},
\end{aligned}
$$


and the $\mathscr{O}\left(\delta^{-1}\right)$ contributions to the boundary conditions given by Eqs. (3.20)-(3.22) on $\xi=0$ are, remembering that $\mathbf{n}$ points into the fluid:

$$
\begin{aligned}
\frac{\partial N_{0}^{D}}{\partial \xi}+N_{0}^{D} \frac{\partial \phi_{0}^{D}}{\partial \xi} & =0 \\
\frac{\partial C_{0}^{D}}{\partial \xi}-C_{0}^{D} \frac{\partial \phi_{0}^{D}}{\partial \xi} & =0 \\
-\frac{\partial \phi_{0}^{D}}{\partial \xi} & =Q .
\end{aligned}
$$

Eqs. (3.31)-(3.36) have analytic solution, matching the outer solutions specified by Eq. (3.23), that are given by

$$
\begin{aligned}
\phi_{0}^{D} & =\Phi+2 \log \left(\tanh \left(\sqrt{\frac{\bar{N}}{2}} \xi+\frac{1}{2} \sinh ^{-1} \frac{2 \sqrt{2 \bar{N}}}{(-Q)}\right)\right), \\
N_{0}^{D} & =\bar{N} \exp \left(\Phi-\phi_{0}^{D}\right), \\
C_{0}^{D} & =\bar{N} \exp \left(\phi_{0}^{D}-\Phi\right) .
\end{aligned}
$$

The solutions given by Eq. (3.37)-(3.39) are plotted in Figure 3 for $\bar{N}=1, Q=-1$.

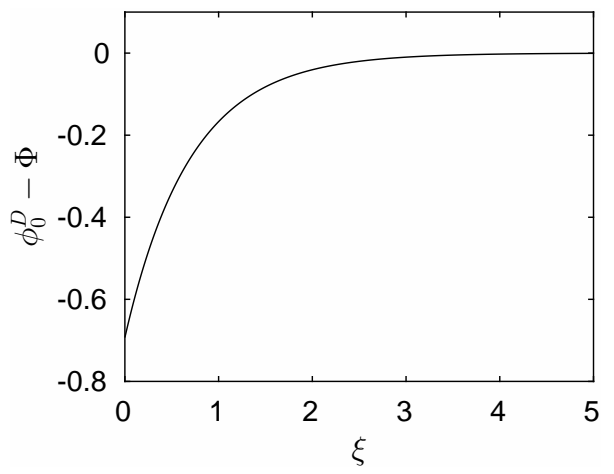

(a)

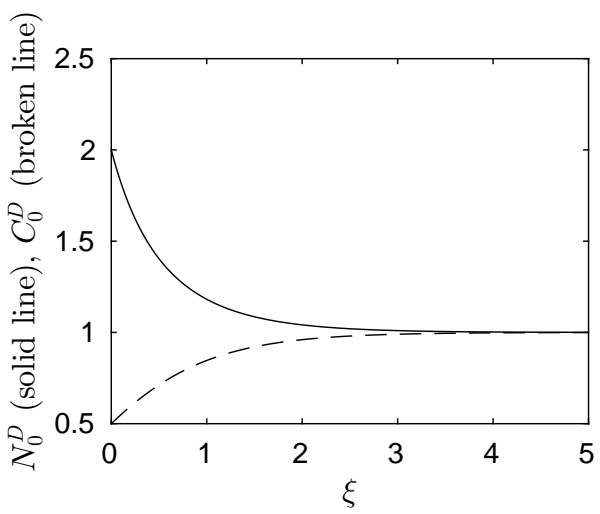

(b)

FIG. 3. (a) The leading order dimensionless potential in the Debye layer given by Eq. (3.37), and (b) the leading order dimensionless sodium ion concentration (solid line) and dimensionless chloride ion concentration (broken line) in the Debye layer given by Eqs. (3.38) and (3.39). Parameter values are $\bar{N}=1, Q=-1$.

The analytic solutions given by Eqs. (3.37)-(3.39) allow us to obtain an analytic solution for the swelling pressure. Using Eqs. (3.31)-(3.32) and (3.34)-(3.35), we may use Eq. (3.15) to write the leading order gradient of the swelling pressure in the Debye layer as

$$
\frac{\partial p_{\mathrm{swell}, 0}^{D}}{\partial \xi}=\mathscr{A} \frac{\partial}{\partial \xi}\left(N_{0}^{D}+C_{0}^{D}\right)
$$


which we may integrate and then deduce, by using Eqs. (3.38) and (3.39) and matching the outer and Debye layer solutions using Eq. (3.24), that

$$
p_{\text {swell }, 0}^{D}-p_{\text {swell }, 0}^{o}=2 \mathscr{A} \bar{N}\left(\cosh \left(\phi_{0}^{D}-\Phi\right)-1\right) .
$$

To estimate the dimensional charge density on the surface of the solid scaffold we write both Eq. (3.37) and (3.40) in dimensional variables:

$$
\begin{array}{r}
\phi_{0}^{D^{*}=\Phi^{*}+2} \frac{R^{*} T^{*}}{F^{*}} \log \left(\tanh \left(\sqrt{\frac{F^{*} \bar{N}^{*}}{2 \varepsilon_{f}^{*} R^{*} T^{*}}} s^{*}+\frac{1}{2} \sinh ^{-1} \frac{2 \sqrt{2 \bar{N}^{*} R^{*} T^{*} \varepsilon_{f}^{*}}}{\left(-Q^{*}\right)}\right)\right), \\
p_{\text {swell }, 0}^{D^{*}}-p_{\text {swell }, 0}^{o^{*}}=2 R^{*} T^{*} \bar{N}^{*}\left(\cosh \left(\frac{F^{*}}{R^{*} T^{*}}\left(\phi_{0}^{D^{*}}-\Phi^{*}\right)\right)-1\right),
\end{array}
$$

where $s^{*}$ is the shortest dimensional distance to the charged surface. The difference in dimensional swelling pressure across the Debye layer, $\Delta p_{\mathrm{swell}}^{*}$, is defined by

$$
\Delta p_{\mathrm{swell}}^{*}=\left.p_{\mathrm{swell}, 0}^{D^{*}}\right|_{s^{*}=0}-p_{\mathrm{swell}, 0}^{o^{*}},
$$

and may be calculated by substituting $s^{*}=0$ into Eq. (3.41), and substituting the resulting value of the dimensional potential into Eq. (3.42). In Figure 4 we plot the relationship between $\Delta p_{\text {swell }}^{*}$ and $Q^{*}$ using the parameters given in Table 1 , and setting $\bar{N}^{*}=\Pi_{0}^{*}$. Noting that atmospheric pressure is around $10^{5} \mathrm{~N} \mathrm{~m}^{-2}$, and that the relationship between dimensional and dimensionless variables in Eq. (3.12) gives $Q \approx 1$ when $\left|Q^{*}\right|=0.03 \mathrm{C} \mathrm{m}^{-2}$, we observe that our analysis predicts that $Q=\mathscr{O}(1)$ when the swelling pressure is comparable to atmospheric pressure. This is what we would expect - swelling pressure is known to make a significant contribution to the deformation of cartilage tissue, and this can only occur if it is comparable in size to other phenomena such as atmospheric pressure. We note that the swelling pressure can cause the pressure in some joints to reach values as high as $1.8 \times 10^{7} \mathrm{~N} \mathrm{~m}^{-2}$ [21].

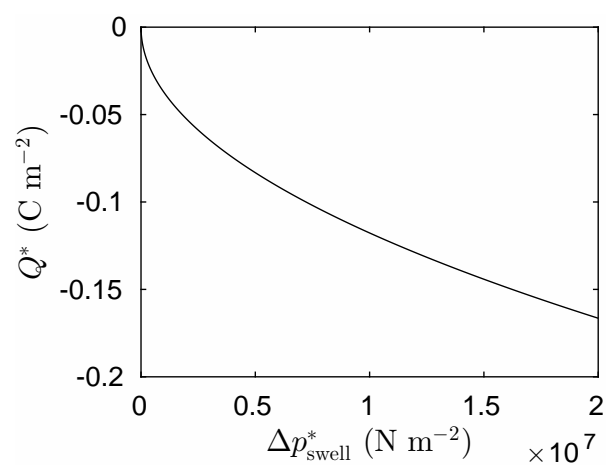

FIG. 4. The relationship between $\Delta_{p \text { swell }}^{*}$ and $Q^{*}$ for the parameters given Table 1 , and setting $\bar{N}^{*}=\Pi_{0}^{*}$. 


\subsection{The Lorentz force induced by the charged interface}

We now write down an expression for the leading order dimensional Lorentz force per unit area, $\mathbf{p}_{\mathbf{L}}{ }^{*}$, that is caused by the charged interface between the fluid and the solid, which will be used when deriving our tissue level poroviscoelastic model. Noting from Eq. (3.37) that the largest contribution to $\nabla_{\mathbf{y}^{*}} \phi^{*}$ on the interface between the fluid and solid is in the direction of $\mathbf{n}$ (which points into the fluid), we approximate $\mathbf{p}_{\mathbf{L}}{ }^{*}$ by

$$
\mathbf{p}_{\mathbf{L}}{ }^{*}=-Q^{*}\left(\mathbf{n} \cdot \nabla_{\mathbf{y}^{*}} \phi^{*}\right) \mathbf{n} .
$$

The boundary condition given by Eq. (3.10) then allows us to write

$$
\mathbf{p}_{\mathbf{L}}^{*}=\frac{Q^{*^{2}}}{\varepsilon^{*}} \mathbf{n}
$$

\section{The poroviscoelastic material}

We now use the leading order solution from Section 3 to write down a coupled fluid-solid interaction problem in the porous microstructure in the case where the scaffold is a viscoelastic material. We write down the governing equations, nondimensionalise these equations, and then construct leading order asymptotic expansions in both the fluid and the solid regions. These leading order asymptotic expansions are then used in a homogenisation of the porous material to give the governing equations at the macroscale.

\subsection{The governing equations}

We write down the governing equations in both the solid and fluid regions, and on the interface between these regions, and then nondimensionalise these equations. Note that the nondimensionalisation of the fluid region is different to that used in Section 3, as we are now interested in different lengthscales.

4.1.1 The fluid region. On using Eqs. (3.28) and (3.30) we see that both the velocity of the fluid, and the sum of the hydrodynamic pressure and swelling pressure, do not vary across the Debye layer. The leading order Cauchy dimensional stress tensor in the fluid therefore has entries given by, for $i, j=$ $1,2,3$ :

$$
\sigma_{i j}^{*}=\frac{\mu_{f}^{*}}{2}\left(\frac{\partial v_{i}^{*}}{\partial y_{j}^{*}}+\frac{\partial v_{j}^{*}}{\partial y_{i}^{*}}\right)-\left(p^{*}+p_{\mathrm{swell}}^{*}\right) \delta_{i j},
$$

where $\mathbf{v}^{*}, p^{*}, p_{\text {swell }}^{*}$ are the leading order dimensional velocity, pressure and swelling pressure consistent with the outer solution derived in Section 3.3, and we remind ourselves that $p_{\text {swell }}^{*}$ is constant away from the Debye layers. The fluid velocity and pressure then satisfy the Navier-Stokes equations [1]: for $\mathbf{y}^{*} \in \Omega_{f}^{*}$ :

$$
\begin{aligned}
\rho_{f}^{*}\left(\frac{\partial v_{i}^{*}}{\partial t^{*}}+v_{j}^{*} \frac{\partial v_{i}^{*}}{\partial y_{j}^{*}}\right) & =-\frac{\partial}{\partial y_{i}^{*}}\left(p^{*}+p_{\mathrm{swell}}^{*}\right)+\mu_{f}^{*} \frac{\partial^{2} v_{i}^{*}}{\partial y_{j}^{* 2}}, \quad i=1,2,3, \\
\frac{\partial v_{i}^{*}}{\partial y_{i}^{*}} & =0 .
\end{aligned}
$$


We use the nondimensionalisation

$$
\mathbf{y}^{*}=d^{*} \mathbf{y}, \quad \mathbf{v}^{*}=V^{*} \mathbf{v}, \quad p^{*}+p_{\mathrm{swell}}^{*}=\frac{\mu_{f}^{*} L^{*} V^{*}}{d^{*^{2}}} p, \quad \sigma^{*}=\frac{\mu_{f}^{*} L^{*} V^{*}}{d^{*^{2}}} \sigma, \quad t^{*}=\frac{d^{*}}{\varepsilon V^{*}} t,
$$

where $d^{*}$ is again the characteristic lengthscale of the microstructure, $L^{*}$ is the characteristic macro lengthscale, $V^{*}$ is representative of the fluid velocity, the swelling pressure has been absorbed into the dimensionless pressure, the separation of scales parameter $\varepsilon$ is defined by

$$
\varepsilon=\frac{d^{*}}{L^{*}}
$$

and we assume that $\varepsilon \ll 1$. We remark that $\varepsilon_{f}^{*}$ has been used for the permittivity in Section 3 , and $\varepsilon$ is not related to the permittivity, but is instead a small parameter. Although acknowledging the potential for confusion we believe that using conventional symbols for these quantities makes the presentation clearer, but would like to emphasise that $\varepsilon_{f}^{*}$ and $\varepsilon$ do actually represent different entities.

The dimensionless Cauchy stress tensor in the fluid region is given by, for $i, j=1,2,3$ :

$$
\sigma_{i j}=\frac{\varepsilon}{2}\left(\frac{\partial v_{i}}{\partial y_{j}}+\frac{\partial v_{j}}{\partial y_{i}}\right)-p \delta_{i j}
$$

and the dimensionless governing equations in the fluid region are then given by, for $\mathbf{x} \in \Omega_{f}$ :

$$
\begin{aligned}
\varepsilon^{2} \operatorname{Re}\left(\varepsilon \frac{\partial v_{i}}{\partial t}+v_{j} \frac{\partial v_{i}}{\partial y_{j}}\right) & =-\frac{\partial p}{\partial y_{i}}+\varepsilon \frac{\partial^{2} v_{i}}{\partial y_{j}^{2}}, \quad i=1,2,3 \\
\frac{\partial v_{i}}{\partial y_{i}} & =0
\end{aligned}
$$

where $\operatorname{Re}=\rho_{f}^{*} V^{*} L^{*} / \mu_{f}^{*}$ is the macroscale Reynolds number. The scalings are application specific; we remark that other authors, [22, 39, 44], use slightly different scalings.

4.1.2 The solid region. We denote the first Piola-Kirchhoff tensor by $S^{*}$. The indices in this tensor are ordered so that the force per unit undeformed area in the $i$ th coordinate direction, on a surface in the undeformed body with normal vector $\mathbf{N}$, is given by $S_{M i}^{*} N_{M}$. The dimensional equations for incompressible, nonlinearly viscoelastic deformations in the solid region are given by [24], for $\mathbf{Y}^{*} \in$ $\Omega_{s, 0}^{*}$ :

$$
\begin{aligned}
\rho_{s} \frac{\partial^{2} y_{i}^{*}}{\partial t^{*^{2}}} & =\frac{\partial S_{M i}^{*}}{\partial Y_{M}^{*}}, \quad i=1,2,3 \\
\operatorname{det}(G) & =1,
\end{aligned}
$$

where $S^{*}$ may be written

$$
S_{M i}^{*}=\hat{S}_{M i}^{*}\left(G, \dot{G}^{*}\right)-\operatorname{det}(G) G_{M i}^{-1} q^{*}, \quad i, M=1,2,3,
$$

$\rho_{s}$ is the density of the scaffold, $\hat{S}^{*}$ is the dimensional first Piola-Kirchhoff stress tensor in the absence of the incompressibility constraint, $q^{*}$ is the dimensional Lagrange multiplier used to enforce the incompressibility constraint, $G$ is the deformation gradient tensor of the solid, with entries given by

$$
G_{i M}=\frac{\partial y_{i}^{*}}{\partial Y_{M}^{*}}, \quad i, M=1,2,3,
$$


and $\dot{G}^{*}$ is the derivative with respect to dimensional time of the deformation gradient tensor. We have not used a superscript asterisk on $G$; this is because it is already dimensionless. It is more usual to use $F$ to represent the deformation gradient tensor. We use $G$ to use the deformation gradient tensor in the solid region (which is not defined in the fluid region), and will reserve $F$ for use later as a deformation gradient tensor at the macroscale that applies to the whole body being modelled. Note that we could use Eq. (4.8) to remove the dependence of Eq. (4.9) on $\operatorname{det}(G)$. We persist with this term as it will be required in the later analysis, for example the derivation of Eq. (4.12) below.

A nondimensionalisation, consistent with that used in the fluid region, is

$$
\mathbf{Y}^{*}=d^{*} \mathbf{Y}, \quad \mathbf{y}^{*}=d^{*} \mathbf{y}, \quad q^{*}=\frac{\mu_{f}^{*} L^{*} V^{*}}{d^{*^{2}}} q, \quad \hat{S}^{*}=\frac{\mu_{f}^{*} L^{*} V^{*}}{d^{*^{2}}} \hat{S}, \quad t^{*}=\frac{d^{*}}{\varepsilon V^{*}} t .
$$

The deformation gradient tensor and its inverse are given in terms of dimensionless coordinates by

$$
G_{i M}=\frac{\partial y_{i}}{\partial Y_{M}}, \quad G_{M i}^{-1}=\frac{\partial Y_{M}}{\partial y_{i}}, \quad i, M=1,2,3 .
$$

We write the dimensionless first Piola-Kirchhoff stress tensor in the solid region as, for $i, M=1,2,3$ :

$$
S_{M i}=\hat{S}_{M i}-\operatorname{det}(G) G_{M i}^{-1} q .
$$

Using the identity

$$
\frac{\partial}{\partial Y_{M}}\left(\operatorname{det}(G) G_{M i}^{-1}\right)=0, \quad i=1,2,3
$$

the dimensionless governing equations in the solid region are given by, for $\mathbf{Y} \in \Omega_{s, 0}$ :

$$
\begin{aligned}
\varepsilon^{4} \frac{\rho_{s}}{\rho_{f}} \operatorname{Re} \frac{\partial^{2} y_{i}}{\partial t^{2}} & =\frac{\partial \hat{S}_{M i}}{\partial Y_{M}}-\operatorname{det}(G) G_{M i}^{-1} \frac{\partial q}{\partial Y_{M}}, \quad i=1,2,3 \\
\operatorname{det} G & =1
\end{aligned}
$$

4.1.3 The interface between the solid and the fluid. We have written the governing equations in the fluid phase using an Eulerian coordinate system, and the governing equations in the solid phase using a Lagrangian coordinate system. The conditions on the interface between these two regions will therefore contain quantities from different coordinate systems, and care must be taken to ensure that these conditions are correct.

The first dimensional interface condition is continuity of velocity across the interface, i.e. we apply a no-slip boundary condition between the fluid and the solid. Let a point $\tilde{\mathbf{Y}}^{*} \in \partial \Omega_{f s, 0}^{*}$ on the interface in the Lagrangian frame be mapped to $\tilde{\mathbf{y}}^{*} \in \partial \Omega_{f s}^{*}$ on the interface in the Eulerian frame. Equating the velocity on either side of the interface gives

$$
\left.\mathbf{v}^{*}\right|_{\tilde{\mathbf{y}}^{*}}=\left.\frac{\partial \mathbf{y}^{*}}{\partial t^{*}}\right|_{\tilde{\mathbf{Y}}^{*}}
$$

The nondimensionalisation given by Eqs. (4.3) and (4.10) then gives the following dimensionless interface condition:

$$
\left.\mathbf{v}\right|_{\tilde{\mathbf{y}}}=\left.\varepsilon \frac{\partial \mathbf{y}}{\partial t}\right|_{\tilde{\mathbf{Y}}}
$$


The second interface condition is continuity of stress across the interface. Aside from the stress due to the Cauchy stress tensor in the fluid given by Eq. (4.4) and the first Piola-Kirchoff stress tensor in the solid given by Eq. (4.11), we also have to consider the Lorentz force induced by the charged surface of the interface, given by Eq. (3.43). Using the following nondimensionalisation, consistent with that used in both the fluid and solid regions:

$$
\mathbf{p}_{\mathbf{L}}^{*}=\frac{\mu_{f}^{*} L^{*} V^{*}}{d^{* 2}} \mathbf{p}_{\mathbf{L}}, \quad Q^{*}=\frac{\sqrt{\mu_{f}^{*} L^{*} V^{*} \varepsilon_{f}^{*}}}{d^{*}} Q,
$$

the dimensionless Lorentz force is given by

$$
\mathbf{p}_{\mathbf{L}}=Q^{2} \mathbf{n} .
$$

After transforming the Cauchy stress tensors to the first Piola-Kirchhoff stress tensor on the interface (where $G$ is defined), we use Eqs. (4.4), (4.11) and (4.16) to deduce that continuity of stress on the interface gives, for $\mathbf{Y}^{*} \in \partial \Omega_{f s, 0}^{*}$ :

$$
\left(S_{M i}+\operatorname{det}(G) G_{M j}^{-1}\left(Q^{2} \delta_{i j}-\sigma_{i j}\right)\right) N_{M}=0, \quad i=1,2,3,
$$

where $\mathbf{N}$ is the unit normal vector to the interface in the Lagrangian coordinate system, pointing into the fluid. We will also require Eq. (4.17) in the Eulerian frame; this is given by

$$
\left(\tau_{i j}+Q^{2} \delta_{i j}-\sigma_{i j}\right) n_{j}=0, \quad i=1,2,3
$$

where $\mathbf{n}$ is the unit normal vector to the interface in the Eulerian coordinate system, pointing into the fluid, and $\tau=(1 / \operatorname{det} G) G S$ is the Cauchy stress tensor in the solid phase.

\subsection{Multiple scales analysis}

We assume that the microstructure in the Lagrangian frame consists of repeating units $\hat{\Omega}_{0}$, comprising a solid region $\hat{\Omega}_{s, 0}$, a fluid region $\hat{\Omega}_{f, 0}$, and a fluid solid interface $\partial \hat{\Omega}_{f s, 0}$. The corresponding regions in the Eulerian coordinate system are $\hat{\Omega}, \hat{\Omega}_{s}, \hat{\Omega}_{f}$ and $\partial \hat{\Omega}_{f s}$. The repeating unit $\hat{\Omega}_{0}$ is unchanged across tissue. The solid region, fluid region and interface between these regions are permitted to vary slowly across tissue to allow us to capture the effects of macroscale variations in both porosity of the tissue and charge density on the interface between the fluid and solid phases.

We introduce a macroscale Lagrangian coordinate $\mathbf{X}$ that is related to the microscale coordinate by $\mathbf{X}=\varepsilon \mathbf{Y}$. With the standard assumption of multiple scales analyses that the macro- and the micro-scale may be treated as independent of one another in generating asymptotically valid solutions, the gradient operator becomes

$$
\nabla_{\mathbf{Y}} \rightarrow \nabla_{\mathbf{Y}}+\varepsilon \nabla_{\mathbf{X}}
$$

We assume all solutions are periodic in the microscale coordinate $\mathbf{Y}$. In the fluid we write

$$
\begin{aligned}
v_{i} & =v_{i}^{0}+\varepsilon v_{i}^{1}+\varepsilon^{2} v_{i}^{2}+\ldots \\
p & =p^{0}+\varepsilon p^{1}+\varepsilon^{2} p^{2}+\ldots \\
\sigma_{i j} & =\sigma_{i j}^{0}+\varepsilon \sigma_{i j}^{1}+\varepsilon^{2} \sigma_{i j}^{2}+\ldots \\
& =-p^{0} \delta_{i j}+\varepsilon\left(\frac{\partial v_{i}^{0}}{\partial y_{j}}+\frac{\partial v_{j}^{0}}{\partial y_{i}}-p^{1} \delta_{i j}\right)+\varepsilon^{2}\left(\frac{\partial v_{i}^{1}}{\partial y_{j}}+\frac{\partial v_{j}^{1}}{\partial y_{i}}+\frac{\partial v_{i}^{0}}{\partial x_{j}}+\frac{\partial v_{j}^{0}}{\partial x_{i}}-p^{2} \delta_{i j}\right)+\ldots
\end{aligned}
$$


and in the solid we define a macroscale coordinate by $\mathbf{x}=\varepsilon \mathbf{y}$ and denote the velocity of a point in the solid region by $\mathbf{v}^{s}$ :

$$
\begin{aligned}
x_{i} & =x_{i}^{0}+\varepsilon x_{i}^{1}+\varepsilon^{2} x_{i}^{2}+\ldots \\
y_{i} & =\frac{1}{\varepsilon} x_{i}^{0}+x_{i}^{1}+\varepsilon^{1} x_{i}^{2}+\ldots \\
v_{i}^{s} & =\varepsilon \frac{\partial y_{i}}{\partial t} \\
& =\frac{\partial x_{i}}{\partial t} \\
& =v_{i}^{s, 0}+\varepsilon v_{i}^{s, 1}+\varepsilon^{2} v_{i}^{s, 2}+\ldots \\
G_{i M} & =G_{i M}^{0}+\varepsilon G_{i M}^{1}+\varepsilon^{2} G_{i M}^{2}+\ldots \\
& =\frac{1}{\varepsilon} \frac{\partial x_{i}^{0}}{\partial Y_{M}}+\left(\frac{\partial x_{i}^{0}}{\partial X_{M}}+\frac{\partial x_{i}^{1}}{\partial Y_{M}}\right)+\varepsilon\left(\frac{\partial x_{i}^{1}}{\partial X_{M}}+\frac{\partial x_{i}^{2}}{\partial Y_{M}}\right)+\ldots \\
\dot{G}_{i M} & =\dot{G}_{i M}^{0}+\varepsilon \dot{G}_{i M}^{1}+\varepsilon^{2} \dot{G}_{i M}^{2}+\ldots \\
& =\frac{1}{\varepsilon} \frac{\partial^{2} x_{i}^{0}}{\partial t \partial Y_{M}}+\left(\frac{\partial^{2} x_{i}^{0}}{\partial t \partial X_{M}}+\frac{\partial^{2} x_{i}^{1}}{\partial t \partial Y_{M}}\right)+\varepsilon\left(\frac{\partial^{2} x_{i}^{1}}{\partial t \partial X_{M}}+\frac{\partial^{2} x_{i}^{2}}{\partial t \partial Y_{M}}\right)+\ldots \\
\hat{S}_{M i} & =\hat{S}_{M i}^{0}+\varepsilon \hat{S}_{M i}^{1}+\varepsilon^{2} \hat{S}_{M i}^{2}+\ldots \\
q & =q^{0}+\varepsilon q^{1}+\varepsilon^{2} q^{2}+\ldots \\
\tau_{i j} & =\tau_{i j}^{0}+\varepsilon \tau_{i j}^{1}+\varepsilon^{2} \tau_{i j}^{2}+\ldots
\end{aligned}
$$

We will also make the assumption that $Q$ varies slowly across tissue, allowing us to write $Q=Q(\mathbf{X})$.

The multiple scales formulation above is suitable when the differential equations are posed in Lagrangian coordinates. In some of the later analysis it will be convenient to write the differential equations in Eulerian coordinates. The independent coordinates are then the microscale Eulerian coordinate $\mathbf{y}$, and the macroscale Eulerian coordinate $\mathbf{x}=\varepsilon \mathbf{y}$. The gradient operator becomes

$$
\nabla_{\mathbf{y}} \rightarrow \nabla_{\mathbf{y}}+\varepsilon \nabla_{\mathbf{x}} .
$$

In the following sections we will make it clear whether we are working in the Eulerian or Lagrangian frame, allowing the reader to deduce from context whether the Eulerian coordinates are the solution of the problem, or the independent coordinates.

As the solid region $\hat{\Omega}_{s}$ and fluid region $\hat{\Omega}_{f}$ within the repeating unit that we homogenise over in Eulerian coordinates vary slowly across the tissue, it is important to note that the normal vector to the interface between these regions also varies slowly across the tissue. One approach that has been used to allow the normal vector to retain dependency on both the microscale and the macroscale coordinates is to use Reynold's transport theorem as described by Holmes [22]; see, for example, [7, 40, 41]. An alternative approach $[6,12]$, which we adopt here, is to use the method of level sets by defining a function $\chi$ such that $\chi(\mathbf{y}, \mathbf{x})<0$ in the solid, and $\chi(\mathbf{y}, \mathbf{x})>0$ in the fluid. The interface is then defined by the level set $\chi(\mathbf{y}, \mathbf{x})=0$. The normal to the interface is then given by the gradient of $\chi$; we remark that the sign of $\chi$ in the solid and fluid regions ensure that the gradient of $\chi$ points out of the solid region into the fluid region. We also define $\chi$ so that when $\chi$ is independent of $\mathbf{x}$ the normal vector to the interface, of unit magnitude, is given by

$$
\mathbf{n}=\nabla_{\mathbf{y}} \chi
$$


i.e. $\chi$ is scaled so that the gradient of $\chi$ with respect to the microscale coordinate on the interface between the fluid and the solid is of unit magnitude. When $\chi=\chi(\mathbf{y}, \mathbf{x})$, the normal vector pointing out of the solid region into the fluid region, of unit magnitude, is given by

$$
\mathbf{n}=\frac{\nabla_{\mathbf{y}} \chi+\varepsilon \nabla_{\mathbf{x}} \chi}{\left\|\nabla_{\mathbf{y}} \chi+\varepsilon \nabla_{\mathbf{x}} \chi\right\|}
$$

and so

$$
\begin{aligned}
\left\|\nabla_{\mathbf{y}} \chi+\varepsilon \nabla_{\mathbf{x}} \chi\right\| \mathbf{n} & =\mathbf{n}^{0}+\varepsilon \nabla_{\mathbf{x}} \chi \\
\text { where } \quad \mathbf{n}^{0} & =\nabla_{\mathbf{y}} \chi .
\end{aligned}
$$

Similarly, we could define a corresponding function $\chi_{0}$ in Lagrangian coordinates such that $\mathbf{N}$ is given by

$$
\begin{aligned}
\left\|\nabla_{\mathbf{Y}} \chi_{0}+\varepsilon \nabla_{\mathbf{X}} \chi_{0}\right\| \mathbf{N} & =\mathbf{N}^{0}+\varepsilon \nabla_{\mathbf{X}} \chi_{0} \\
\text { where } \quad \mathbf{N}^{0} & =\nabla_{\mathbf{Y}} \chi_{0}
\end{aligned}
$$

In practical applications the normal vector $\mathbf{n}=\left(n_{1}, n_{2}, n_{3}\right)^{\top}$, with unit magnitude, may be specified at each point on the interface between the fluid and the solid in the microscale coordinate system, rather than the function $\chi$. We may then define $\chi$ locally at any point $\mathbf{y}_{0}=\left(y_{1}^{0}, y_{2}^{0}, y_{3}^{0}\right)^{\top}$ on the interface by

$$
\chi=n_{1}\left(y_{1}-y_{1}^{0}\right)+n_{2}\left(y_{2}-y_{2}^{0}\right)+n_{3}\left(y_{3}-y_{3}^{0}\right) \text {. }
$$

This definition of $\chi$ satisfies both Eq. (4.19) and the condition that $\chi=0$ on the interface. A similar approach could be used to specify a local definition of $\chi_{0}$ in the vicinity of the interface in Lagrangian coordinates.

We note that although the Reynold's transport theorem approach and the level set approach described above give identical results for macroscopically uniform media, these two approaches have not yet been proved to coincide in the general case.

4.2.1 An initial result from the fluid region. The $\mathscr{O}(1)$ contribution to Eq. (4.5) allows us to write

$$
-\frac{\partial p^{0}}{\partial y_{i}}=0, \quad \mathbf{y} \in \Omega_{f}, \quad i=1,2,3
$$

from which we may deduce that $p^{0}$ is independent of the microscale coordinate, and write

$$
p^{0}=p^{0}(\mathbf{X}, t) .
$$

This result will be used when deriving the asymptotic solution in the solid region in Section 4.2.2 which, in turn, will be required when deriving the asymptotic solution in the fluid region in Section 4.2.3.

4.2.2 The solid region. We assume that the leading order entries of $S$ are of the same order as the leading order entries of $G$. The $\mathscr{O}\left(\varepsilon^{-1}\right)$ contributions to Eqs. (4.12) and (4.17) then allow us to deduce that

$$
\mathbf{x}^{0}=\mathbf{x}(\mathbf{X}, t)
$$


and so the leading order macroscale deformation is independent of the microscale coordinates. The leading order macroscale deformation gradient tensor $F=F(\mathbf{X})$ is then defined by

$$
F_{i M}=\frac{\partial x_{i}^{0}}{\partial X_{M}}, \quad i, M=1,2,3
$$

The $\mathscr{O}(1)$ contributions to Eqs. (4.12) and (4.13) are, for $\mathbf{Y} \in \hat{\Omega}_{s, 0}$ :

$$
\begin{aligned}
\frac{\partial \hat{S}_{M i}^{0}}{\partial Y_{M}}-\operatorname{det}\left(G^{0}\right)\left(G^{0}\right)_{M i}^{-1} \frac{\partial q^{0}}{\partial Y_{M}} & =0, \quad i=1,2,3 \\
\operatorname{det}\left(G^{0}\right) & =1,
\end{aligned}
$$

where

$$
\hat{S}_{M i}^{0}=\hat{S}_{M i}\left(G^{0}, \dot{G}^{0}\right)
$$

On using Eq. (4.4), the $\mathscr{O}(1)$ contribution to the interface condition given by Eq. (4.17) is

$$
\left(\hat{S}_{M i}^{0}-\operatorname{det}\left(G^{0}\right)\left(G^{0}\right)_{M i}^{-1}\left(q^{0}-p^{0}-Q^{2}\right)\right) N_{M}=0 .
$$

We saw in Eq. (4.21) that $p^{0}$ is independent of the microscale coordinate $\mathbf{Y}$. We have also assumed that $Q=Q(\mathbf{X})$. Writing

$$
q^{0}=p^{0}+Q^{2}+\tilde{q}^{0}
$$

Eqs. (4.24), (4.25) and (4.26) become, for $\mathbf{Y} \in \hat{\Omega}_{s, 0}$ :

$$
\begin{aligned}
\frac{\partial \hat{S}_{M i}^{0}}{\partial Y_{M}}-\operatorname{det}\left(G^{0}\right)\left(G^{0}\right)_{M i}^{-1} \frac{\partial \tilde{q}^{0}}{\partial Y_{M}} & =0, \quad i=1,2,3 \\
\operatorname{det}\left(G^{0}\right) & =1,
\end{aligned}
$$

with boundary condition for $\mathbf{Y} \in \hat{\Omega}_{f s, 0}$ :

$$
\left(\hat{S}_{M i}^{0}-\operatorname{det}\left(G^{0}\right)\left(G^{0}\right)_{M i}^{-1} \tilde{q}^{0}\right) N_{M}=0, \quad i=1,2,3 .
$$

The solution to the differential equations and boundary conditions given by Eqs. (4.27), (4.28) and (4.29) is independent of $p^{0}$ and $Q^{2}$ and so we may write

$$
\tilde{q}^{0}=\tilde{q}^{0}\left(\mathbf{Y}, G^{0}, \dot{G}^{0}\right) .
$$

The leading order stress tensor is then given by, for $i, M=1,2,3$ :

$$
S_{M i}^{0}=\tilde{S}_{M i}^{0}\left(\mathbf{Y}, G^{0}, \dot{G}^{0}\right)-\operatorname{det}\left(G^{0}\right)\left(G^{0}\right)_{M i}^{-1}\left(p^{0}(\mathbf{X})+Q^{2}(\mathbf{X})\right), \quad i, M=1,2,3,
$$

where, using our observation encapsulated by Eq. (4.30), $\tilde{S}_{M i}^{0}$ is periodic in $\mathbf{Y}$, and is given by

$$
\tilde{S}_{M i}^{0}\left(\mathbf{Y}, G^{0}, \dot{G}^{0}\right)=\hat{S}_{M i}^{0}\left(G^{0}, \dot{G}^{0}\right)-\operatorname{det}\left(G^{0}\right)\left(G^{0}\right)_{M i}^{-1} \tilde{q}_{0}\left(\mathbf{Y}, G^{0}, \dot{G}^{0}\right), \quad i . M=1,2,3 .
$$

It is worth commenting on the structure of the leading order first Piola-Kirchhoff stress tensor given by Eq. (4.31). $p^{0}$ and $Q$ appear only through the sum $p^{0}+Q^{2}$, and this sum plays the role that would be played by the Lagrangian multiplier that enforces incompressibility of the scaffold in the absence of a charge on the surface of the scaffold. 
4.2.3 The fluid region. The analysis in this section is based closely on that presented in [39]. We have already demonstrated (see Eq. (4.21)) that the leading order pressure is independent of the microscale coordinate. Using Eq. (4.4) we may write

$$
p^{0}=p^{0}(\mathbf{x}, t), \quad \sigma_{i j}^{0}=-\delta_{i j} p^{0}(\mathbf{x}, t) .
$$

The $\mathscr{O}(\varepsilon)$ contribution to Eq. (4.5) and the $\mathscr{O}(1)$ contribution to Eq. (4.6) give

$$
\begin{aligned}
\frac{\partial^{2} v_{i}^{0}}{\partial y_{j}^{2}}-\frac{\partial p^{1}}{\partial y_{i}} & =\frac{\partial p^{0}}{\partial x_{i}}, \quad i=1,2,3 \\
\frac{\partial v_{i}^{0}}{\partial y_{i}} & =0
\end{aligned}
$$

and the leading order contribution to the interface condition given by Eq. (4.15) is

$$
v_{i}^{0}=\frac{\partial x_{i}^{0}}{\partial t}, \quad i=1,2,3
$$

The structure of Eqs. (4.33)-(4.36) suggests that the solutions for $\mathbf{v}^{0}$ and $p^{1}$ take the form

$$
v_{i}^{0}=\frac{\partial x_{i}^{0}}{\partial t}-K_{i j}(\mathbf{y}, \mathbf{x}, t) \frac{\partial p^{0}}{\partial x_{j}}, \quad p^{1}=-a_{i}(\mathbf{y}, \mathbf{x}, t) \frac{\partial p^{0}}{\partial x_{i}}+\tilde{p}^{1}(\mathbf{x}, t),
$$

where both $K_{i j}$ and $a_{i}$ are periodic in $\mathbf{y}$ for $i, j=1,2,3$. Remembering from Eq. (4.22) that $x_{i}^{0}$ is independent of $\mathbf{y}$, and using Eqs. (4.34)-(4.37) we obtain, for $\mathbf{y} \in \hat{\Omega}_{f}$,

$$
\begin{aligned}
\left(\frac{\partial^{2} K_{i k}}{\partial y_{j}^{2}}-\frac{\partial a_{k}}{\partial y_{i}}+\delta_{i k}\right) \frac{\partial p^{0}}{\partial x_{k}} & =0, \quad i=1,2,3, \\
\frac{\partial K_{i k}}{\partial y_{i}} \frac{\partial p^{0}}{\partial x_{k}} & =0,
\end{aligned}
$$

together with the boundary condition that for $\mathbf{y} \in \partial \hat{\Omega}_{f s}$ :

$$
K_{i k} \frac{\partial p^{0}}{\partial x_{k}}=0, \quad i=1,2,3 .
$$

We now derive a system of equations for $K_{i j}, i, j=1,2,3$ that are independent of $p^{0}$. Eq. (4.38) implies that

$$
\frac{\partial^{2} K_{i k}}{\partial y_{j}^{2}}-\frac{\partial a_{k}}{\partial y_{i}}+\delta_{i k}=M_{i k}(\mathbf{x})
$$

where

$$
M_{i k} \frac{\partial p^{0}}{\partial x_{k}}=0, \quad i=1,2,3
$$

Writing

$$
f_{k}(\mathbf{x}, \mathbf{y})=M_{i k}(\mathbf{x}) y_{i}, \quad k=1,2,3,
$$


we see that we can absorb $\mathbf{f}$ into $\mathbf{a}$ and write

$$
\frac{\partial^{2} K_{i k}}{\partial y_{j}^{2}}-\frac{\partial a_{k}}{\partial y_{i}}=-\delta_{i k}, \quad i, k=1,2,3 .
$$

Similarly, Eq. (4.39) implies that for $\mathbf{y} \in \hat{\Omega}_{f}$ :

$$
\frac{\partial K_{i k}}{\partial y_{i}}=g_{k}(\mathbf{x}), \quad k=1,2,3
$$

where

$$
g_{k} \frac{\partial p^{0}}{\partial x_{k}}=0
$$

and Eq. (4.40) implies that, for $\mathbf{y} \in \partial \hat{\Omega}_{f s}$ :

$$
K_{i k}=h_{i k}(\mathbf{x}), \quad i, k=1,2,3,
$$

where

$$
h_{i k} \frac{\partial p^{0}}{\partial x_{k}}=0, \quad i=1,2,3 .
$$

Based on the arguments given in Eqs. (4.41)-(4.46), the most general form for $K_{i k}$ is then

$$
K_{i k}=K_{i k}^{0}+h_{i k}(\mathbf{x})+y_{i} g_{k}(\mathbf{x}), \quad i, k=1,2,3,
$$

with, for $\mathbf{y} \in \partial \hat{\Omega}_{f s}$ :

$$
K_{i k}^{0}=0 .
$$

Due to Eqs. (4.44) and (4.46) we see that $h_{i k}$ and $g_{k}$ do not affect the velocity given by Eq. (4.37). Without loss of generality we may then set $\mathbf{g}=\mathbf{0}$ and $h_{i k}=0$ and write Eq. (4.43) as, for $\mathbf{y} \in \hat{\Omega}_{f}$ :

$$
\frac{\partial K_{i k}}{\partial y_{i}}=0, \quad k=1,2,3 .
$$

On using Eq. (4.45) the boundary condition given by Eq. (4.40) may be written, for $\mathbf{y} \in \partial \hat{\Omega}_{f s}$ :

$$
K_{i k}=0, \quad i, k=1,2,3 .
$$

Eqs. (4.42) and (4.47), together with the boundary condition given by Eq. (4.48) give a Stokes type problem for each $k=1,2,3$, where $K_{i k}$ is the $i$ 'th component of the 'velocity', and $a_{k}$ is the 'pressure', and we remark that $K_{i j}$ and $a_{i}$ depend only on the deformed microstructure through the region, $\hat{\Omega}_{f}$, on which the system of equations are defined. Noting $\mathbf{a}$ is unique up to a function of $\mathbf{x}$, analogous to pressure being unique up to a constant in Stokes flow with velocity boundary conditions, it is subsequently fixed uniquely by demanding that

$$
\int_{\hat{\Omega}_{f}} p^{1} \mathrm{~d} V_{f}=0
$$

where we write $\mathrm{d} V_{f}$ to emphasise the region we are integrating over. In particular, this constraint forces the microdomain mean of the leading order pressure solution to be accurate to at least second order in the perturbation expansion. 


\subsection{Derivation of the macroscale equations}

We now combine the results from Sections 4.2.1, 4.2.2 and 4.2.3 to derive the governing equations at the macroscale. We define

$$
J=\operatorname{det}(F),
$$

where $F$ is the macroscale deformation gradient tensor defined by Eq. (4.23), and note that

$$
F_{M i}^{-1}=\frac{\partial X_{M}}{\partial x_{i}^{0}}
$$

Remembering that $\mathbf{x}^{0}=\mathbf{x}^{0}(\mathbf{X})$ we see that $F, F^{-1}$ and $J$ are independent of the microscale coordinate. We will also need to define the volume fraction of both the solid and fluid regions, in both Lagrangian and Eulerian coordinates, when deriving the macroscale equations. These are given by

$$
\theta_{s}=\frac{\left|\hat{\Omega}_{s}\right|}{|\hat{\Omega}|}, \quad \theta_{f}=\frac{\left|\hat{\Omega}_{f}\right|}{|\hat{\Omega}|}, \quad \theta_{s, 0}=\frac{\left|\hat{\Omega}_{s, 0}\right|}{\left|\hat{\Omega}_{0}\right|}, \quad \theta_{f, 0}=\frac{\left|\hat{\Omega}_{f, 0}\right|}{\left|\hat{\Omega}_{0}\right|} .
$$

The volume fraction of the fluid phase is also known as the porosity. The change of variables between Lagrangian and Eulerian coordinates allows us to write

$$
\left|\hat{\Omega}_{s}\right|+\left|\hat{\Omega}_{f}\right|=|\hat{\Omega}|=J(\mathbf{X}, \mathbf{t})\left|\hat{\Omega}_{0}\right|=J\left(\left|\hat{\Omega}_{s, 0}\right|+\left|\hat{\Omega}_{f, 0}\right|\right) .
$$

As the solid is incompressible at the microscale level we have

$$
\begin{aligned}
\left|\hat{\Omega}_{s}\right| & =\left|\hat{\Omega}_{s, 0}\right|, \\
\left|\hat{\Omega}_{f}\right| & =\frac{J-\theta_{s, 0}}{1-\theta_{s, 0}}\left|\hat{\Omega}_{f, 0}\right|, \\
\theta_{s} & =\frac{\theta_{s, 0}}{J}, \\
\theta_{f} & =\frac{J-\theta_{s, 0}}{J} .
\end{aligned}
$$

We will require a transport theorem when deriving the macroscale equations to give an expression for the rate of change, with respect to a macroscale coordinate, of an integral over a domain at the microscale, where the domain varies spatially at the macroscale level. For a function $f^{s}$ defined on $\hat{\Omega}_{s}$, that is periodic in the microscale coordinate $\mathbf{y}$, the transport theorem gives $[6,12]$ :

$$
\frac{\partial}{\partial x_{i}} \int_{\hat{\Omega}_{s}} f^{s}(\mathbf{x}, \mathbf{y}) \mathrm{d} V_{s}=\int_{\hat{\Omega}_{s}} \frac{\partial f^{s}}{\partial x_{i}} \mathrm{~d} V_{s}-\int_{\partial \hat{\Omega}_{f s}} f^{s} \frac{\partial \chi}{\partial x_{i}} \mathrm{~d} S_{f s},
$$

where $\chi(\mathbf{x}, \mathbf{y})=0$ is the level set that defines the interface between the fluid and solid phase, discussed in Section 4.2. The first term on the right hand side of Eq. (4.49) takes account of the rate of change, with respect to the macroscale coordinate, of the function being integrated. The second term takes account of the rate of change of the domain, with respect to the macroscale coordinate. Similarly, for a function $f^{f}$ defined on $\hat{\Omega}_{f}$, that is periodic in the microscale coordinate $\mathbf{y}$, the transport theorem gives

$$
\frac{\partial}{\partial x_{i}} \int_{\hat{\Omega}_{f}} f^{f}(\mathbf{x}, \mathbf{y}) \mathrm{d} V_{f}=\int_{\hat{\Omega}_{f}} \frac{\partial f^{f}}{\partial x_{i}} \mathrm{~d} V_{f}+\int_{\partial \hat{\Omega}_{f s}} f^{f} \frac{\partial \chi}{\partial x_{i}} \mathrm{~d} S_{f s},
$$

where the difference in sign on the second term on the right hand side compared to Eq. (4.49) arises because $\mathbf{n}$ defined by Eq. (4.20) points out of the region occupied by the solid, and into the region occupied by the fluid. 
4.3.1 Conservation of mass. We now derive the macroscale equation that corresponds to conservation of mass. The $\mathscr{O}(\varepsilon)$ contribution to Eq. (4.6) is

$$
\frac{\partial v_{i}^{1}}{\partial y_{i}}+\frac{\partial v_{i}^{0}}{\partial x_{i}}=0, \quad \mathbf{y} \in \hat{\Omega}_{f}
$$

Integrating over $\hat{\Omega}_{f}$, applying the divergence theorem, and remembering that: (i) $\mathbf{n}$ points into $\hat{\Omega}_{f}$; and (ii) $\mathbf{v}^{1}$ is periodic in the microscale coordinate, we obtain

$$
-\int_{\partial \hat{\Omega}_{f s}} v_{i}^{1} n_{i} \mathrm{~d} S_{f s}+\int_{\hat{\Omega}_{f}} \frac{\partial v_{i}^{0}}{\partial x_{i}} \mathrm{~d} V_{f}=0 .
$$

Neglecting terms of $\mathscr{O}(\varepsilon)$ in the normal vector $\mathbf{n}$ defined by Eq. (4.20), which generates only $\mathscr{O}\left(\varepsilon^{2}\right)$ error, application of the transport theorem given by Eq. (4.50) yields

$$
-\int_{\partial \hat{\Omega}_{f s}} v_{i}^{1} n_{i}^{0} \mathrm{~d} S_{f s}+\frac{\partial}{\partial x_{i}} \int_{\hat{\Omega}_{f}} v_{i}^{0} \mathrm{~d} V_{f}-\int_{\partial \hat{\Omega}_{f s}} v_{i}^{0} \frac{\partial \chi}{\partial x_{i}} \mathrm{~d} S_{f s}=0 .
$$

To obtain the corresponding equation for the solid phase we invoke Euler's identity (see, for example, [1]):

$$
\frac{\mathrm{d}}{\mathrm{d} t}(\operatorname{det}(G))=\operatorname{det}(G) \frac{\partial v_{i}^{s}}{\partial y_{i}}
$$

where $\mathbf{v}^{s}$ is the velocity of the solid phase. This allows us to write the $\mathscr{O}(\varepsilon)$ contribution to Eq. (4.13) in Eulerian coordinates as

$$
\frac{\partial v_{i}^{s, 1}}{\partial y_{i}}+\frac{\partial v_{i}^{s, 0}}{\partial x_{i}}=0, \quad \mathbf{y} \in \hat{\Omega}_{s}
$$

and using a simlar argument to that used in the fluid region we obtain

$$
\int_{\partial \hat{\Omega}_{f s}} v_{i}^{s, 1} n_{i}^{0} \mathrm{~d} S_{f s}+\frac{\partial}{\partial x_{i}} \int_{\hat{\Omega}_{s}} v_{i}^{s, 0} \mathrm{~d} V_{s}+\int_{\partial \hat{\Omega}_{f s}} v_{i}^{s, 0} \frac{\partial \chi}{\partial x_{i}} \mathrm{~d} S_{f s}=0 .
$$

Defining the average velocity in the fluid and solid phases by

$$
\bar{v}_{i}^{0}=\frac{1}{\left|\hat{\Omega}_{f}\right|} \int_{\hat{\Omega}_{f}} v_{i}^{0} \mathrm{~d} V_{f}, \quad \bar{v}_{i}^{s, 0}=\frac{1}{\left|\hat{\Omega}_{s}\right|} \int_{\hat{\Omega}_{s}} v_{i}^{s, 0} \mathrm{~d} V_{s}, \quad i=1,2,3,
$$

we may use the interface condition Eq. (4.15), and Eqs. (4.51) and (4.52), to give conservation of mass in Eulerian coordinates at leading order:

$$
\frac{\partial}{\partial x_{i}}\left(\theta_{f} \bar{v}_{i}^{0}+\theta_{s} \bar{v}_{i}^{s, 0}\right)=0 .
$$

The expression for $v_{i}^{0}$ given by Eq. (4.37) allows us to deduce, on switching to Lagrangian coordinates where $v_{i}^{s, 0}=\partial x_{i}^{0} / \partial t$, that

$$
J F_{M i}^{-1} \frac{\partial}{\partial X_{M}}\left(\frac{\partial x_{i}^{0}}{\partial t}-\bar{K}_{i j} F_{N j}^{-1} \frac{\partial p^{0}}{\partial X_{N}}\right)=0
$$


where

$$
\bar{K}_{i j}=\frac{1}{|\hat{\Omega}|} \int_{\hat{\Omega}_{f}} K_{i j} \mathrm{~d} V_{f} .
$$

Finally, on using the identities

$$
\begin{aligned}
\frac{\partial J}{\partial F_{i M}} & =J F_{M i}^{-1}, & i . M & =1,2,3, \\
\frac{\partial}{\partial X_{M}}\left(J F_{M i}^{-1}\right) & =0, & i & =1,2,3,
\end{aligned}
$$

we may write Eq. (4.54) as

$$
\frac{\partial J}{\partial t}=\frac{\partial}{\partial X_{M}}\left(J F_{M i}^{-1} \bar{K}_{i j} F_{N j}^{-1} \frac{\partial p^{0}}{\partial X_{N}}\right) .
$$

We may also write down an expression for the leading order fluid flux. Using Eqs. (4.37), (4.53) and (4.55) we see that the leading order fluid flux per unit deformed area, $\mathscr{F}^{f}$, is given by, for $i=1,2,3$ :

$$
\begin{aligned}
\mathscr{F}_{i}^{f} & =\theta_{f} \bar{v}_{i}^{0} \\
& =\theta_{f} \frac{\partial x_{i}^{0}}{\partial t}-\bar{K}_{i j} F_{N j}^{-1} \frac{\partial p^{0}}{\partial X_{N}} .
\end{aligned}
$$

4.3.2 Conservation of momentum. We now derive an equation that corresponds to conservation of momentum at the macroscale. Writing Eq. (4.12) in Eulerian coordinates, at $\mathscr{O}(\varepsilon)$, Eqs. (4.5) and (4.12) yield

$$
\begin{array}{ll}
\frac{\partial \sigma_{i j}^{1}}{\partial y_{j}}+\frac{\partial \sigma_{i j}^{0}}{\partial x_{j}}=0, & \mathbf{x} \in \hat{\Omega}_{f},
\end{array}
$$

and Eq. (4.31) gives

$$
\tau_{i j}^{0}=\hat{\tau}_{i j}^{0}-\left(p^{0}(\mathbf{X})+Q^{2}(\mathbf{X})\right) \delta_{i j}, \quad i, j=1,2,3,
$$

where

$$
\hat{\tau}_{i j}^{0}=\frac{1}{\operatorname{det}\left(G^{0}\right)} \tilde{S}_{M i}^{0} G_{j M}^{0}=\tilde{S}_{M i}^{0} G_{j M}^{0}, \quad i, j=1,2,3,
$$

and $\tilde{S}$ is defined by Eq. (4.32). Integrating over the appropriate domain, applying the divergence theorem, remembering that $\mathbf{n}$ points from $\hat{\Omega}_{s}$ into $\hat{\Omega}_{f}$, and noting that the solutions are periodic in $\mathbf{y}$ yields

$$
\begin{array}{rr}
-\int_{\partial \hat{\Omega}_{f s}} \sigma_{i j}^{1} n_{j} \mathrm{~d} S_{f s}+\int_{\hat{\Omega}_{f}} \frac{\partial \sigma_{i j}^{0}}{\partial x_{j}} \mathrm{~d} V_{f}=0, & i=1,2,3, \\
\int_{\partial \hat{\Omega}_{f s}} \tau_{i j}^{1} n_{j} \mathrm{~d} S_{f s}+\int_{\hat{\Omega}_{s}} \frac{\partial \tau_{i j}^{0}}{\partial x_{j}} \mathrm{~d} V_{s}=0 . & i=1,2,3 .
\end{array}
$$


Using Eq. (4.20) to neglect terms of $\mathscr{O}(\varepsilon)$ in $\mathbf{n}$, which only induces errors at $\mathscr{O}\left(\varepsilon^{2}\right)$, and applying the transport theorem given by Eqs. (4.49) and (4.50), allows us to write Eqs. (4.62) and (4.63) as

$$
\begin{aligned}
-\int_{\partial \hat{\Omega}_{f s}} \sigma_{i j}^{1} n_{j}^{0} \mathrm{~d} S_{f s}+\frac{\partial}{\partial x_{j}} \int_{\hat{\Omega}_{f}} \sigma_{i j}^{0} \mathrm{~d} V_{f}-\int_{\hat{\Omega}_{f s}} \sigma_{i j}^{0} \frac{\partial \chi}{\partial x_{j}} \mathrm{~d} S_{f s}=0, & i=1,2,3, \\
\int_{\partial \hat{\Omega}_{f s}} \tau_{i j}^{1} n_{j}^{0} \mathrm{~d} S_{f s}+\frac{\partial}{\partial x_{j}} \int_{\hat{\Omega}_{s}} \tau_{i j}^{0} \mathrm{~d} V_{s}+\int_{\hat{\Omega}_{f s}} \tau_{i j}^{0} \frac{\partial \chi}{\partial x_{j}} \mathrm{~d} S_{f s}=0 . & i=1,2,3 .
\end{aligned}
$$

The $\mathscr{O}(\varepsilon)$ contribution to Eq. (4.18) gives, on using Eq. (4.20),

$$
\left(\tau_{i j}^{0}-\sigma_{i j}^{0}-Q^{2} \delta_{i j}\right) \frac{\partial \chi}{\partial x_{j}}+\left(\tau_{i j}^{1}-\sigma_{i j}^{1}\right) n_{j}^{0}=0, \quad i=1,2,3 .
$$

Furthermore, the divergence theorem allows us to write

$$
\int_{\hat{\Omega}_{f s}} n_{i} \mathrm{~d} S_{f s}=0 .
$$

Taking the $\mathscr{O}(\varepsilon)$ contribution to Eq. (4.67), as $Q=Q(\mathbf{X})$ we can write

$$
\int_{\hat{\Omega}_{f s}} Q^{2} \delta_{i j} \frac{\partial \chi}{\partial x_{j}} \mathrm{~d} S_{f s}=0
$$

Combining Eqs. (4.64)-(4.68) gives

$$
\frac{\partial}{\partial x_{j}} \int_{\hat{\Omega}_{f}} \sigma_{i j}^{0} \mathrm{~d} V_{f}+\frac{\partial}{\partial x_{j}} \int_{\hat{\Omega}_{s}} \tau_{i j}^{0} \mathrm{~d} V_{s}=0, \quad i=1,2,3 .
$$

Defining the averaged stress tensors at leading order by

$$
\bar{\sigma}_{i j}^{0}=\frac{1}{\left|\hat{\Omega}_{f}\right|} \int_{\hat{\Omega}_{f}} \sigma_{i j}^{0} \mathrm{~d} V_{f}, \quad \bar{\tau}_{i j}^{0}=\frac{1}{\left|\hat{\Omega}_{s}\right|} \int_{\hat{\Omega}_{s}} \tau_{i j}^{0} \mathrm{~d} V_{s}, \quad i, j=1,2,3,
$$

we may write Eq. (4.69) as

$$
\frac{\partial}{\partial x_{j}}\left(\theta_{f} \bar{\sigma}_{i j}^{0}+\theta_{s} \bar{\tau}_{i j}^{0}\right)=0, \quad i=1,2,3,
$$

which gives a force balance in Eulerian coordinates for the averaged leading order stress tensors. Using Eq. (4.33) and (4.60) we may write Eq. (4.70) as

$$
\frac{\partial}{\partial X_{M}}\left(\theta_{s, 0}\left(\bar{S}_{M i}^{0}-F_{M i}^{-1} Q^{2}\right)-J F_{M i}^{-1} p^{0}\right)=0, \quad i=1,2,3
$$

where $\bar{S}^{0}$ is the averaged first Piola-Kirchhoff stress tensor for the solid phase in the absence of the isotropic terms, with entries given by

$$
\bar{S}_{M i}^{0}=\frac{1}{\left|\hat{\Omega}_{s, 0}\right|} \int_{\hat{\Omega}_{s, 0}} F_{M j}^{-1} \hat{\tau}_{j i}^{0} \mathrm{~d} V_{s, 0}, \quad i, M=1,2,3 .
$$

The first Piola-Kirchhoff stress tensor for the homogenised poroelastic body is then given by

$$
T_{M i}=\theta_{s, 0}\left(\bar{S}_{M i}^{0}-F_{M i}^{-1} Q^{2}\right)-J F_{M i}^{-1} p^{0}, \quad i, M=1,2,3 .
$$




\section{The macroscale equations and boundary conditions}

We now write the governing equations in dimensional form, and will use the dimensional equations in this and subsequent sections. As we are no longer using dimensionless variables we will, for clarity, no longer use superscript asterisks to denote dimensional variables. The leading order governing equations, Eqs. (4.56) and (4.71) are, for $\mathbf{X} \in \Omega_{0}$ :

$$
\begin{aligned}
\frac{\partial}{\partial X_{M}}\left(\theta_{s, 0}\left(\bar{S}_{M i}-F_{M i}^{-1} \frac{Q^{2}}{\varepsilon_{f}}\right)\right) & =J F_{M i}^{-1} \frac{\partial p}{\partial X_{M}}, \quad i=1,2,3 \\
\frac{\partial J}{\partial t} & =\frac{\partial}{\partial X_{M}}\left(J F_{M i}^{-1} \bar{K}_{i j} F_{N j}^{-1} \frac{\partial p}{\partial X_{N}}\right),
\end{aligned}
$$

where $\mathbf{x}=\mathbf{x}(\mathbf{X}, t)$ are the coordinates of the deformed body, $\theta_{s, 0} \bar{S}$ is the first Piola-Kirchhoff stress tensor for the poroelastic body (rather than only the solid component) without the term that models swelling pressure or the contribution from $p$ (and will be specified by a constitutive relationship), $F$ is the macroscale deformation gradient tensor, $J=\operatorname{det}(F), p$ is the pressure (that includes both the hydrodynamic and swelling pressures), $\bar{K}$ is the permeability tensor (given by a constitutive relationship) and $\varepsilon_{f}$ is again the permittivity of the fluid, rather than a small parameter used in the homogenisation procedure. It follows from Eq. (4.57) that the leading order dimensional fluid flux per unit undeformed area is given by

$$
\mathscr{F}_{i}^{f}=\theta_{f} \frac{\partial x_{i}^{0}}{\partial t}-\bar{K}_{i j} F_{M j}^{-1} \frac{\partial p^{0}}{\partial X_{M}}
$$

We now require suitable boundary conditions for the system of partial differential equations given by Eqs. (5.1) and (5.2). Remembering that Eq. (5.1) was derived by appealing to conservation of momentum of the whole body, it is appropriate to apply either a displacement or traction boundary condition in each spatial direction. If the solid phase is given a known displacement on the boundary then a suitable boundary condition for Eq. (5.1) is

$$
\mathbf{x}(\mathbf{X})=\mathbf{x}_{0}, \quad \mathbf{X} \in \partial \Omega_{0} .
$$

If, instead, a given force per unit undeformed area $\mathbf{s}$ is applied to the boundary then a suitable boundary condition for Eq. (5.1) is

$$
\left(\theta_{s, 0}\left(\bar{S}_{M i}-F_{M i}^{-1} \frac{Q^{2}}{\varepsilon_{f}}\right)-J F_{M i}^{-1} p\right) N_{M}=s_{i}, \quad \mathbf{X} \in \partial \Omega_{0}, \quad i=1,2,3 .
$$

We now turn our attention to suitable boundary conditions for Eq. (5.2), which was derived by appealing to conservation of mass. If there is no flux of fluid relative to the scaffold - for example if the boundary is displaced by an impermeable body then, using Eq. (5.3), a suitable boundary condition is

$$
\bar{K}_{i j} F_{M j}^{-1} \frac{\partial p}{\partial X_{M}}=0, \quad \mathbf{X} \in \partial \Omega_{0}, \quad i=1,2,3
$$

Suppose, instead, the scaffold is placed in a bath of stationary fluid with constant isotropic pressure $p_{0}$. The isotropic pressure $p_{0}$ will be a sum of both the hydrostatic pressure, and the swelling pressure due to dissolved ions in the bath. A suitable boundary condition for Eq. (5.2) is then

$$
p=p_{0}, \quad \mathbf{X} \in \partial \Omega_{0}
$$




\subsection{Constitutive relations}

To close the macroscale equations we require a constitutive relation for both the permeability tensor and the stress tensor.

5.1.1 The permeability tensor. In Section 4.2.3 we defined $K_{i j}, i, j=1,2,3$, to be the solution, defined in the fluid region of the deformed microstructure, of Eqs. (4.42) and (4.47), together with the boundary condition given by Eq. (4.48). The macroscale permeability tensor $\bar{K}$ is then the average of $K$ defined by Eq. (4.55). Calculation of $\bar{K}$ therefore requires knowledge of the deformed microstructure. This deformation is, however, lost from the model when the microstructure is homogenised. As a consequence, the permeability is usually specified through a constitutive relationship.

Should imaging data representing the microstructure be of a sufficiently high quality then Eqs. (4.42), (4.47), (4.48) and (4.55) may be solved numerically to calculate the permeability tensor of the undeformed body, or of the body under a given deformation. Alternatively, it may be possible to estimate the entries of this tensor experimentally under a range of macroscale deformations, using parameter estimation from observed flux profiles under controlled conditions. It is unlikely, however, that a general permeability tensor, valid for all microstructure deformations, could be generated. To the best of our knowledge no work exists on estimating the deformation of the microstructure that would be required when calculating the permeability tensor under a given deformation.

In the simulations carried out later in this study the permeability tensor is assumed to be isotropic. The simplest choice would be to take $\bar{K}$ to be a constant multiple of the identity matrix. This, however, has the drawback that the permeability is independent of the porosity of the deformed body, and would not restrict fluid flow should the porosity of the deformed body become very small due to deformation of the scaffold restricting flow. To avoid this drawback the permeability tensor is modelled as being dependent on the porosity. We use the Kozeny-Carman formula (see, for example, [33]):

$$
\begin{aligned}
\bar{K} & =k_{0} \frac{\left(1-\theta_{f, 0}\right)^{2}}{\theta_{f, 0}^{3}} \frac{\theta_{f}^{3}}{\left(1-\theta_{f}\right)^{2}} I \\
& =k_{0} \frac{\left(J-\left(1-\theta_{f, 0}\right)\right)^{3}}{J \theta_{f, 0}^{3}} I,
\end{aligned}
$$

where $k_{0}$ is the isotropic permeability of the undeformed scaffold, and $I$ is the identity matrix.

5.1.2 The stress tensor. We will assume that the body is poroviscoelastic. Using a Voigt model of viscoelasticity, we first require an expression for $\bar{S}^{(e)}$, the elastic contribution to $\bar{S}$, for which we assume hyperelasticity and thus the existence of a strain energy function $W$ such that

$$
\bar{S}_{M i}^{(e)}=\frac{\partial W}{\partial F_{i M}} .
$$

We assume further that $\bar{S}^{(e)}$ may be decomposed into the sum of volumetric and distortional (or isochoric) components $\bar{S}^{(e, v)}$ and $\bar{S}^{(e, d)}$ so that $\bar{S}^{(e)}=\bar{S}^{(e, v)}+\bar{S}^{(e, d)}$. Assuming that there are $N_{\text {relax }}$ relaxation processes, and that $\tau_{\gamma}$ is the relaxation time associated with relaxation process $\gamma$, we may then write the entries of $\bar{S}$ as, $[15,17,23,24]$ :

$$
\bar{S}_{M i}=\bar{S}_{M i}^{(e)}+\sum_{\gamma=1}^{N_{\text {relax }}} Q_{M i}^{\gamma}, \quad i, M=1,2,3
$$


where, for $\gamma=1,2, \ldots, N_{\text {relax }}$,

$$
\frac{\mathrm{d} Q_{M i}^{\gamma}}{\mathrm{d} t}+\frac{1}{\tau_{\gamma}} Q_{M i}^{\gamma}=\bar{G} \frac{\mathrm{d} S_{M i}^{(e, d)}}{\mathrm{d} t}, \quad i, M=1,2,3,
$$

for a given constant $\bar{G}$. We note that setting $\bar{G}=0$ results in a completely elastic deformation. The use of $Q_{M i}^{\gamma}$ above is a slight abuse of notation as $Q$ has already been used to represent the charge density on the surface of the scaffold. However we believe that use of familiar notation will result in a clearer exposition, and persist with denoting the viscoelastic contributions to the stress tensor by $Q_{M i}^{\gamma}$. The context in which the notation is used should make it clear which quantity is being referred to.

\subsection{Example solutions}

We now present some solutions to Eqs. (5.1)-(5.2), subject to suitable boundary conditions. We use the Kozeny-Carman porosity tensor given by Eq. (5.8), and a strain energy function, suitable for compressible materials [46], given by

$$
W=\frac{\mu}{2}\left(I_{1}-3\right)+\frac{3 \lambda+2 \mu}{6}(\operatorname{det}(F)-1)^{2},
$$

where $\lambda, \mu$ are the Lamé coefficients, and $I_{1}=\operatorname{trace}\left(F^{\top} F\right)$. This gives

$$
\bar{S}_{M i}^{(e)}=\mu F_{i M}+\frac{3 \lambda+2 \mu}{3} \operatorname{det}(F)(\operatorname{det}(F)-1) F_{M i}^{-1}, \quad i, M=1,2,3 .
$$

We note that $\bar{S}_{M i}^{(e)}$ defined in this way does not give zero stress for the undeformed body, when $F=I$. Noting that

$$
\frac{\partial}{\partial X_{M}}\left(J F_{M i}^{-1}\right)=0, \quad i=1,2,3,
$$

we see that we can add any multiple of $J F^{-1}$ to $\bar{S}^{(e)}$ without affecting Eq. (5.1). We therefore use

$$
\bar{S}_{M i}^{(e)}=\mu\left(F_{i M}-J F_{M i}^{-1}\right)+\frac{3 \lambda+2 \mu}{3} J(J-1) F_{M i}^{-1}, \quad i, M=1,2,3,
$$

which does indeed give zero stress when $F=I$, and decompose $\bar{S}_{M i}^{(e)}$ into

$$
\bar{S}_{M i}^{(e)}=\bar{S}_{M i}^{(e, d)}+\bar{S}_{M i}^{(e, v)}, \quad i, M=1,2,3,
$$

where

$$
\begin{aligned}
& \bar{S}_{M i}^{(e, d)}=\mu\left(F_{i M}-J F_{M i}^{-1}\right), \quad i, M=1,2,3, \\
& \bar{S}_{M i}^{(e, v)}=\frac{3 \lambda+2 \mu}{3} J(J-1) F_{M i}^{-1}, \quad i, M=1,2,3 .
\end{aligned}
$$

The volumetric contribution to Eq. (5.13), given by Eq. (5.15), is a consedquence of $\theta_{s, 0} \bar{S}$ being one of the contributions to the stress tensor for the poroelastic body. This body is compressible, as the volume of fluid may vary due to deformation of the (incompressible) solid component of the poroelastic body. 
We use parameter values taken from [15]: a Young's modulus of $6.3 \times 10^{5} \mathrm{~N} \mathrm{~m}^{-2}$, Poisson ratio of 0.1 , and a permeability in the undeformed body, $k_{0}$ in Eq. (5.8), of $1.72 \times 10^{-15} \mathrm{~m}^{4} \mathrm{~N}^{-1} \mathrm{~s}^{-1}$. The Young's modulus and Poisson ratio yield $\lambda=7.16 \times 10^{4} \mathrm{~N} \mathrm{~m}^{-2}, \mu=2.86 \times 10^{5} \mathrm{~N} \mathrm{~m}^{-2}$. There were three viscoelastic relaxation processes, with relaxation times equally spaced on a logarithmic scale between $0.62 \mathrm{~s}$ and $85 \mathrm{~s}$, and $\bar{G}=5.32$.

In the simulations that follow we investigate the effect of the Debye layer within the pores by comparing the model behaviour with the absence of fixed charge and thus no Debye layers, whereby $Q=0$, to simulations with non-zero $Q$, and we then proceed to investigate the impact of fixed charge heterogeneity.

5.2.1 Steady state, isotropic deformation of homogeneous tissue. Our first example solution is a steady state, isotropic deformation of homogeneous tissue, with $F=\alpha I$ for some constant $\alpha$, where the tissue is inside a bath with pressure $p_{0}$. In the steady state Eqs. (5.9)-(5.11) allow us to deduce that $\bar{S}=\bar{S}^{(e)}$. Using Eq. (5.12) we see that in one dimension we have

$$
\bar{S}_{11}=\frac{3 \lambda+5 \mu}{3}(\alpha-1),
$$

whilst in three dimensions we have

$$
\bar{S}_{M i}=\left(\frac{\mu}{3} \alpha(\alpha-1)\left(2 \alpha^{3}+2 \alpha^{2}+2 \alpha-3\right)+\lambda \alpha^{2}\left(\alpha^{3}-1\right)\right) \delta_{i M}, \quad i, M=1,2,3 .
$$

The solution of Eq. (5.2), subject to boundary conditions given by Eq. (5.7), is

$$
p=p_{0}
$$

Using Eq. (5.5) we see that in one dimension $\alpha$ satisfies

$$
\frac{3 \lambda+5 \mu}{3}(\alpha-1)=\frac{Q^{2}}{\alpha \varepsilon}
$$

whilst in three dimensions we have

$$
\left(\frac{\mu}{3} \alpha(\alpha-1)\left(2 \alpha^{3}+2 \alpha^{2}+2 \alpha-3\right)+\lambda \alpha^{2}\left(\alpha^{3}-1\right)\right)=\frac{Q^{2}}{\alpha \varepsilon} .
$$

In Figure 5 we plot the value of $\alpha$ induced by a charge $Q$ for the parameters given in Table 1 and Section 5.2. The solid line represents the value of $\alpha$ given by Eq. (5.16) in one dimension, and the broken line represents the value of $\alpha$ given by Eq. (5.17) in three dimensions. This gives the expected response - the tissue is undeformed (with $\alpha=1$ ) for zero charge, and the tissue then swells, with $\alpha$ increasing as the magnitude of the negative charge increases.

5.2.2 Confined compression of cartilage tissue with constant $Q$. We now consider the confined compression of cartilage tissue described in [15]. A cylindrical undeformed sample of cartilage tissue, of height $H_{0}$ and radius $R$, occupies the region $0<X_{1}<H_{0}, X_{2}^{2}+X_{3}^{2}<R^{2}$. Throughout the experiment there is zero displacement and no flow of fluid on the boundaries given by $X_{1}=0$ and $X_{2}^{2}+X_{3}^{2}=R^{2}$. The sample is compressed so that the undeformed surface $X_{1}=H_{0}$ is displaced to $x_{1}=0.9 H_{0}$ and held at this 


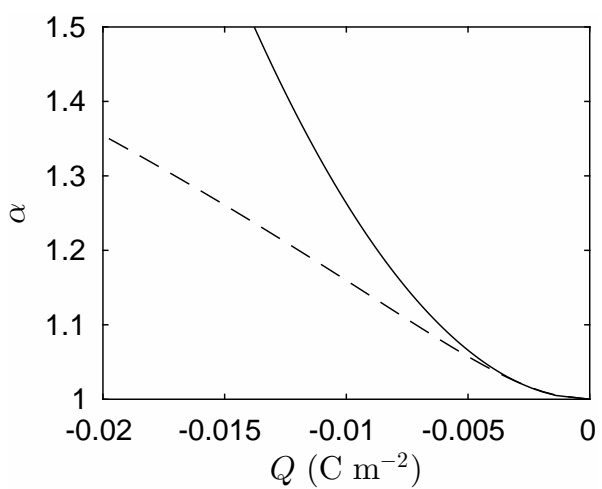

FIG. 5. The stretch factor $\alpha$ as a function of charge $Q$ predicted by Eq. (5.16) and Eq. (5.17) for steady state isotropic deformation of homogeneous tissue discussed in Section 5.2.1. The solid line represents the value of $\alpha$ calculated in one dimension using Eq. (5.16), and the broken line represents the value of $\alpha$ calculated in three dimensions using Eq. (5.17).

displacement until the reaction force per unit area at $X_{1}=H_{0}$ in the direction of the axis of the cylinder reaches a constant value. At time $t=0$ the tissue is then compressed further so that the undeformed surface $X_{1}=H_{0}$ is displaced at a constant rate to $x_{1}=0.85 H_{0}$ over a period of 50 seconds and held at this displacement. Throughout the compression fluid is allowed to flow across the boundary given by $X_{1}=H_{0}$. We define $F(t)$ to be the reaction force per unit area in the direction of the axis of the cylinder at the top of the sample at time $t$.

Aside from specifying the parameter values that appear in Eqs. (5.1) and (5.2) to be those given in Table 1 and Section 5.2, we also need to specify both the charge density $Q$ and scaffold volume fraction $\theta_{s, 0}$. We will investigate the effect of different values of $Q$, and follow [28] in writing

$$
\theta_{s, 0}=0.3-0.15 \frac{X_{1}}{H_{0}}
$$

Suitable boundary conditions for Eq. (5.1) are displacement boundary conditions of the form given by Eq. (5.4) on all boundaries. We apply no-flux boundary conditions for Eq. (5.2) on $X_{1}=0$ and $X_{2}^{2}+X_{3}^{2}=R^{2}$ of the form given by Eq. (5.6), specify the pressure on $X_{1}=H_{0}$ using a boundary condition of the form Eq. (5.7), and seek a solution $x_{1}=x_{1}\left(X_{1}, t\right)$.

We use $H_{0}=1.28 \times 10^{-3} \mathrm{~m}$, as used by [28]. In Figure 6(a) we plot the reaction force per unit area at the top of the cylinder at the end of the initial equilibrium phase as a function of $Q$, and in Figure 6(b) we plot $F(t)-F(0)$, i.e. the additional reaction force per unit area at the top of the cylinder above that seen in the initial equilibrium phase, for: $Q=0$ (solid line); $Q=-0.01 \mathrm{C} \mathrm{m}^{-2}$ (broken line); $Q=-0.02 \mathrm{C} \mathrm{m}^{-2}$ (dot-dashed line); and $Q=-0.03 \mathrm{C} \mathrm{m}^{-2}$ (dotted line). We have used a logarithmic scale for the time axis in Figure 6(b) as is standard when plotting the results of confined compression experiments $[11,15,16,17]$, and note the qualitative similarity of the results in these publications to our results even though they use different mathematical models and parameter values. We see that increasing the magnitude of $Q$ increases: (i) the force per unit area at the top of the cylinder at the end of the initial equilibrium phase; (ii) the magnitude of the difference between the maximum force per unit area above the initial equilibrium value; and (iii) the value of the force per unit area at the final equilibrium value above the initial equilibrium value. 


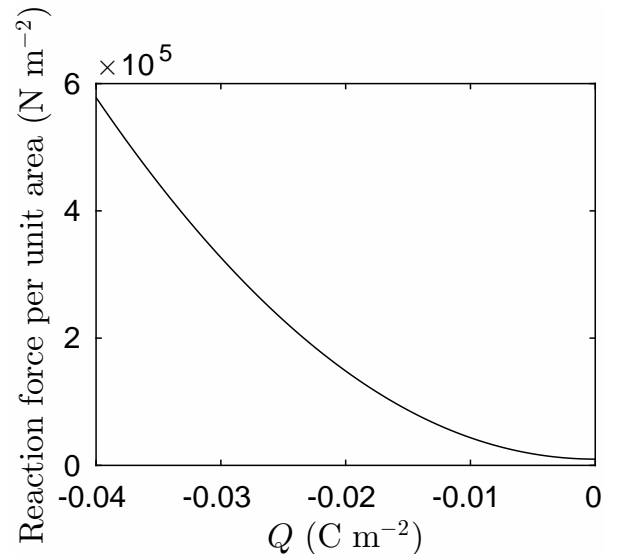

(a)

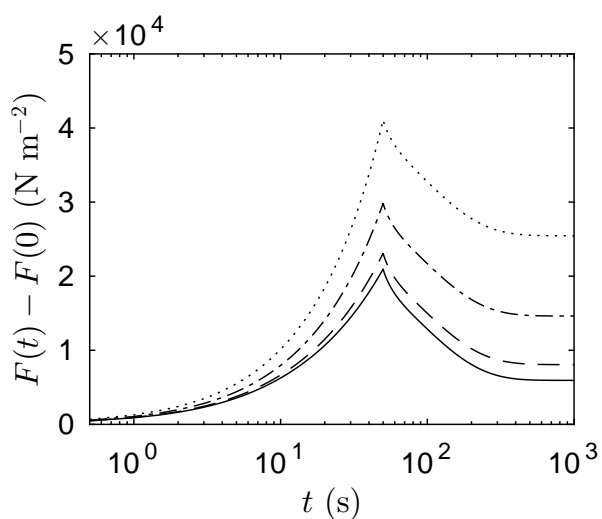

(b)

FIG. 6. (a) The reaction force per unit area as a function of $Q$ at the end of the initial equilibrium phase of the confined compression described in Section 5.2.2. (b) The reaction force per unit area above that seen in the initial equilibrium stage of confined compression. The solid line corresponds to $Q=0$; the broken line to $Q=-0.01 \mathrm{C} \mathrm{m}^{-2}$; the dot-dashed line to $Q=-0.02 \mathrm{C} \mathrm{m}{ }^{-2}$; and the dotted line to $Q=-0.03 \mathrm{C} \mathrm{m}^{-2}$.

5.2.3 Confined compression of cartilage tissue with spatially varying $Q$. We now investigate the effects of an inhomogeneous charge density $Q$ on the output of a confined compression simulation. We allow $Q$ to vary according to

$$
Q\left(X_{1}\right)=Q_{0}+\frac{Q_{1}-Q_{0}}{2}\left(\tanh \left(100\left(\frac{X_{B_{1}}-X_{1}}{H_{0}}\right)\right)+\tanh \left(100\left(\frac{X_{1}-X_{B_{0}}}{H_{0}}\right)\right)\right),
$$

where $Q_{0}=-0.04 \mathrm{C} \mathrm{m}^{-2}$, and $Q_{1}=-0.03 \mathrm{C} \mathrm{m}^{-2}$ and $X_{B_{0}}, X_{B_{1}}$ are given constants. We consider three sets of values of $X_{B_{0}}$ and $X_{B_{1}}$ : (i) $X_{B_{0}}=0.1, X_{B_{1}}=0.4$ simulating charge loss towards the bottom of the sample; (ii) $X_{B_{0}}=0.35, X_{B_{1}}=0.65$ simulating charge loss in the middle of the sample; and (iii) $X_{B_{0}}=0.6, X_{B_{1}}=0.9$ simulating charge loss towards the top of the sample. Plots of $Q$ against $X$ for these values of $X_{B_{0}}$ and $X_{B_{1}}$ are given in Figure 7.

In Figure 8 we plot the force per unit area at the top of the cartilage over that exerted in the initial equilibrium stage. The upper dot-dashed line corresponds to a constant value $Q=-0.04 \mathrm{C} \mathrm{m}^{-2}$, and the lower dot-dashed line to a constant value $Q=-0.03 \mathrm{C} \mathrm{m}^{-2}$. Other lines correspond to $Q$ given by Eq. (5.18), where the solid line corresponds to $X_{B_{0}}=0.1, X_{B_{1}}=0.4$, the broken line to $X_{B_{0}}=0.35, X_{B_{1}}=0.65$, and the dotted line to $X_{B_{0}}=0.6, X_{B_{1}}=0.9$. As would be expected, the force exerted by cartilage where $Q$ is allowed to vary between $0.03 \mathrm{C} \mathrm{m}^{-2}$ and $0.04 \mathrm{C} \mathrm{m}^{-2}$ lies between the force exerted by cartilage with constant $Q=-0.04 \mathrm{C} \mathrm{m}^{-2}$ and the force exerted by cartilage with constant $Q=-0.03 \mathrm{C} \mathrm{m}^{-2}$. We also observe that the force per unit area at the top of the cartilage above that exerted in the initial equilibrium stage is larger when charge loss is located higher in the tissue sample.

In Figure 9(a), (b) and (c) we compare the deformation of cartilage tissue for constant $Q$, where $Q=-0.03 \mathrm{C} \mathrm{m}^{-2}$ and $Q=-0.04 \mathrm{C} \mathrm{m}^{-2}$ (indistinguishable dot-dashed lines), and $Q$ given by Eq. (5.18) (where the solid line corresponds to $X_{B_{0}}=0.1, X_{B_{1}}=0.4$, the broken line to $X_{B_{0}}=0.35, X_{B_{1}}=0.65$, and 


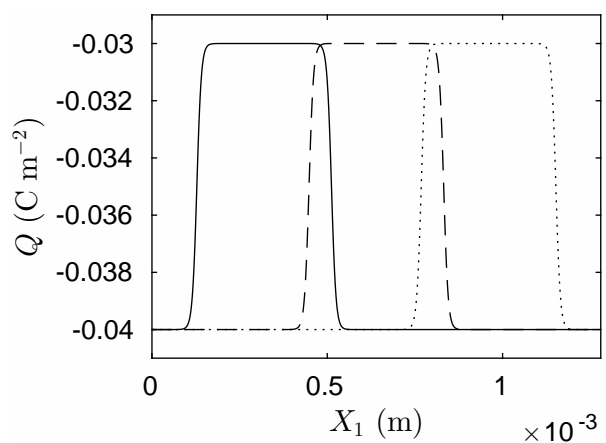

FIG. 7. The spatially varying $Q$ defined by Eq. (5.18), and used in the simulations described in Section 5.2.3. The solid line corresponds to $X_{B_{0}}=0.1, X_{B_{1}}=0.4$, the broken line to $X_{B_{0}}=0.35, X_{B_{1}}=0.65$, and the dotted line to $X_{B_{0}}=0.6, X_{B_{1}}=0.9$.

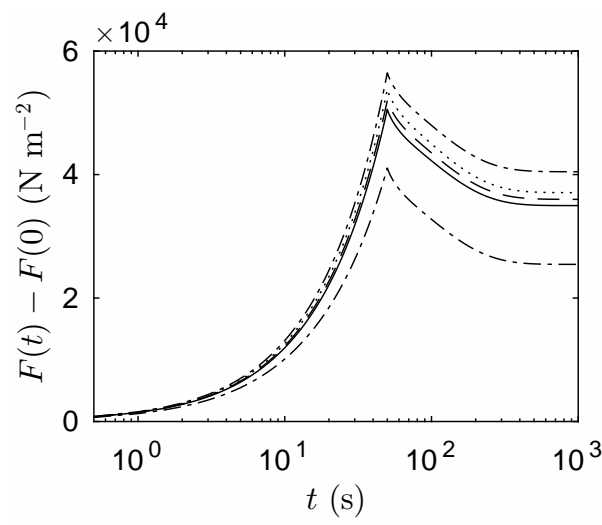

FIG. 8. The reaction force per unit area above that seen in the initial equilibrium stage of the confined compression. The upper dot-dashed line corresponds to a constant value $Q=-0.04 \mathrm{C} \mathrm{m}^{-2}$, and the lower dot-dashed line to a constant value $Q=-0.03 \mathrm{C} \mathrm{m}^{-2}$. Other lines correspond to $Q$ given by Eq. (5.18), where the solid line corresponds to $X_{B_{0}}=0.1, X_{B_{1}}=0.4$, the broken line to $X_{B_{0}}=0.35, X_{B_{1}}=0.65$, and the dotted line to $X_{B_{0}}=0.6, X_{B_{1}}=0.9$.

the dotted line to $X_{B_{0}}=0.6, X_{B_{1}}=0.9$ ), at times $t=0,50,1000 \mathrm{~s}$. In Figure 9(d), (e) and (f) we give the corresponding plots for the deformation gradient $\partial x_{1} / \partial X_{1}$. The confined compression forces at the top of the cartilage tissue predicted by all numerical experiments to be identical. We see that, when $Q$ is allowed to vary, the gradient of the deformation is steeper in spatial regions where the magnitude of $Q$ is higher.

\section{Discussion}

In this manuscript we have systematically developed a homogenisation framework that generates a tissue level poroviscoelastic model for physiological and engineered cartilage even at the scale of large nonlinear deformation, using the nanoscale physics of Debye layer induced swelling pressure together with the porous microstructure of the tissue. The tissue level model is sufficiently general to accommodate 


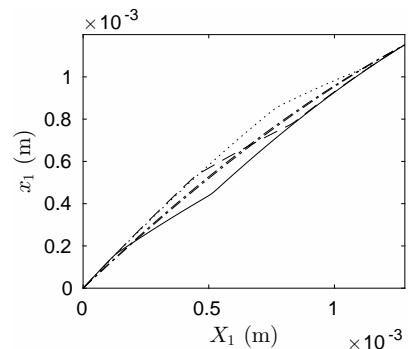

(a) $t=0$

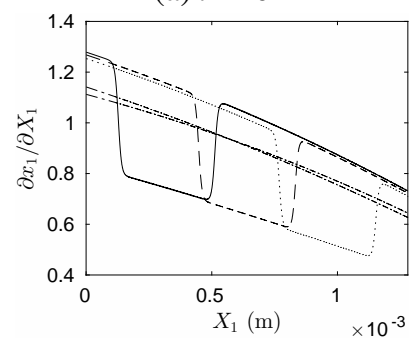

(d) $t=0$

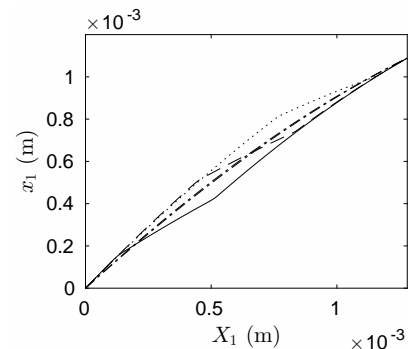

(b) $t=50 \mathrm{~s}$

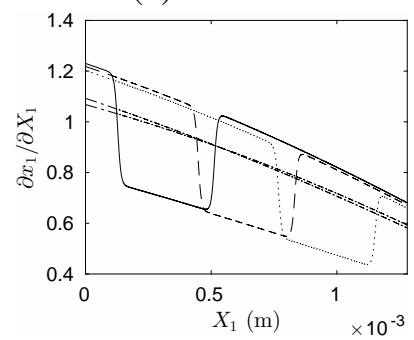

(e) $t=50 \mathrm{~s}$

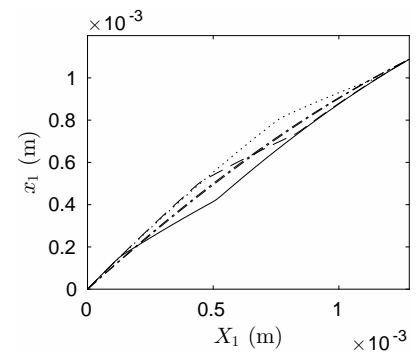

(c) $t=1000 \mathrm{~s}$

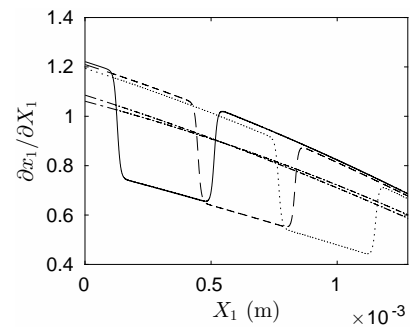

(f) $t=1000 \mathrm{~s}$

FIG. 9. Plots (a), (b), and (c) show the deformed coordinate as a function of the undeformed coordinate at $t=0.50,1000 \mathrm{~s}$ Plots (d), (e) and (f) show the deformation gradient $\partial x_{1} / \partial X_{1}$ as a function of the undeformed coordinate at $t=0.50,1000 \mathrm{~s}$. In all plots indistinguishable dot-dashed lines corresponds to the constant values $Q=-0.03 \mathrm{C} \mathrm{m}^{-2}$ and $Q=-0.04 \mathrm{C} \mathrm{m}{ }^{-2}$. Other lines correspond to $Q$ given by Eq. (5.18), where the solid line corresponds to $X_{B_{0}}=0.1, X_{B_{1}}=0.4$, the broken line to $X_{B_{0}}=0.35, X_{B_{1}}=0.65$, and the dotted line to $X_{B_{0}}=0.6, X_{B_{1}}=0.9$.

complex models of the induction of swelling pressure by fixed charge, beyond the limitations of Donnan theory, as illustrated here with a variant of the Poisson Boltzmann surface charge model devloped by Dean et al. [13] and Han et al. [20]. The framework also provides a rational biophysical derivation of how gradients in porosity, fixed charge density and material properties manifest in tissue level models.

We have illustrated the behaviour of the models generated via this upscaling by considering swelling dynamics, confined compression testing and, especially, the impact of hetereogeneous fixed charge, noting that proteoglycan densities vary spatially in cartilage, with heterogeneous changes accompanying cartilage damage [45]. Firstly, with simple, steady state and isotropic solutions, the homogenised model behaves as expected with swelling increasing with negative fixed charge densities. In the virtual confined compression experiment, performed at a fixed rate of displacement following an initial fixed displacement, one can see from Figure 6 that the tissue is more compliant with reduced magnitudes of homogeneous fixed charge. Together with the qualitative similarity between model behaviour here for confined compression and in previous studies [11, 15, 16, 17], this further demonstrates that the homogenised model is behaving as expected.

Once heterogeneous fixed charge is introduced, as in Figure 7, one can see that the forces required to deform the cartilage in Figure 8 vary with the location of the fixed charge loss. In particular, the homogenised model predicts that the mechanical performance of the cartilage is increasingly compromised with the depth of proteoglycan loss. Comparing Figure 7 with Figure 9 further reveals that the deformation gradient $\partial x_{1} / \partial X_{1}$ is lower compared to homogeneous tissue in localised regions of fixed charge loss and higher elsewhere, though the stress is constant throughout the tissue by Eq. (5.1). Thus 
the homogenised model with heterogeneous fixed charge predicts that the constant stress enforced by the constraints of confined compression is accommodated with a reduced deformation gradient in regions of lower fixed charge, with concomitant larger deformation gradients away from the site of fixed charge loss.

More generally, the cartilage homogenisation framework presented here can be used to develop upscaled models that facilitate the exploration of how ultrasmall length scale biophysics and spatial heterogeneity, whether physiological or localised lesion, impacts cartilage health and dysfunction at the tissue scale, which is accessible to standard diagnostics. In turn such upscaling methods have the potential to contribute to the understanding of the sufficient characteristics required of engineered cartilage substitute and molecular based therapies to ameliorate cartilage dysfunction [20]. These developments are especially timely given smallscale models underpinning cartilage mechanics have been undergoing testing and validation [13], while micro- and nano-scale measurement capabilities are ever increasing $[9,20]$ and cartilage heterogeneities on these scales have already been observed [20]. Such developments suggest that it would be feasible to test hypotheses generated by homogenised models such as the modelling prediction emerging from this study, that a localised fixed charge reduction induces a compensation via increased deformation gradients elsewhere under confined compression. Homogeneised models can also contribute to understanding the functional consequences of the emerging micro- and nano-scale heterogeneities being observed in cartilage, though extensive further theoretical work is required. This includes integrating the approaches here with the homogenisation of cellular inclusions $[18,19]$, and incorporating many further aspects of small-scale cartilage structure within tissue level models, such as the heterogeneous anisotropy of the collagen fibres and more complex, experimentally tested, Poisson Boltzmann models of the induction of swelling pressure via glycoaminoglycan fixed charge.

\section{Acknowledgements}

The authors are grateful to Professor Vaclav Klika of the Czech Technical University in Prague and Professor Cameron Brown of Queensland University of Technology for helpful discussions on cartilage mechanics.

\section{References}

[1] D.J. Acheson. Elementary Fluid Dynamics. Oxford University Press, Oxford, UK, 1990.

[2] N.M. Bachrach, V.C. Mow, and F. Guilak. Incompressibility of the solid matrix of articular cartilage under high hydrostatic pressures. Journal of Biomechanics, 31:445-451, 1998.

[3] C.H. Barnett. Measurement and interpretation of synovial fluid viscosities. Annals of the Rheumatic Diseases, 17:229-233, 1958.

[4] L. Berger, R. Bordas, D. Kay, and S. Tavener. A stabilized finite element method for finite-strain three-field poroelasticity. Computational Mechanics, 60:51-68, 2017.

[5] M.A. Biot. Theory of finite deformations of porous solids. Indiana University Mathematics Journal, 21:597-620, 1972.

[6] M. Bruna and S.J. Chapman. Diffusion in spatially varying porous media. SIAM Journal on Applied mathematics, 75:1648-1674, 2015. 
[7] R. Burridge and J.B. Keller. Poroelasticity equations derived from microstructure. Journal of the Acoustical Society of America, 70:1140-1146, 1981.

[8] M.D. Buschmann and A.J. Grodzinsky. A molecular model of proteoglycan-associated electrostatic forces in cartilage mechanics. Journal of Biomechanical Engineering, 117:179-192, 1995.

[9] P.L. Chandran, E.K. Dimitriadis, E.L. Mertz, and F. Horkay. Microscale mapping of extracellular matrix elasticity of mouse joint cartilage: an approach to extracting bulk elasticity of soft matter with surface roughness. Soft Matter, 14(15):2879-2892, 2018.

[10] D. Chapelle, J.-F. Gerbeau, J. Sainte-Marie, and I.E. Vignon-Clementel. A poroelastic model valid in large strains with applications to perfusion in cardiac modelling. Computational Mechanics, 46:91-101, 2010.

[11] M.J. Chen, L.S. Kimpton, J.P. Whiteley, M. Castilho, J. Malda, C.P. Please, S.L. Waters, and H.M. Byrne. Multiscale modelling and homogenisation of fibre-reinforced hydrogels for tissue engineering. European Journal of Applied Mathematics, 2019.

[12] M.P. Dalwadi, I.M. Griffiths, and M. Bruna. Understanding how porosity gradients can make a better filter using homogenization theory. Proceeings of the Royal Society A, 471:20150464, 2015.

[13] D. Dean, J. Seog, C. Ortiz, and A.J. Grodzinsky. Molecular-level theoretical model for electrostatic interactions within polyelectrolyte brushes: Applications to charged glycosaminoglycans. Langmuir, 19(13):5526-5539, 2003.

[14] I. Demirkan, M.B. Unlu, and B. Bilen. Determining sodium diffusion through acoustic impedance measurements using $80 \mathrm{MHz}$ Scanning Acoustic Microscopy: Agarose phantom verification. Ultrasonics, 94:10-19, 2019.

[15] M.R. DiSilvestro and J-K. Francis Suh. A cross-validation of the biphasic poroviscoelastic model of articular cartilage in uncomfined compression, indentation, and confined compression. Journal of Biomechanics, 34:519-525, 2001.

[16] M.R. DiSilvestro, Q. Zhu, and J-K. Francis Suh. Biphasic poroviscoelastic simulation of the unconfined compression of articular cartilage: II-effect of variable strain rates. Journal of Biomechanical Engineering, 123:198-200, 2001.

[17] M.R. DiSilvestro, Q. Zhu, M. Wong, J.S. Jurvelin, and J-K. Francis Suh. Biphasic poroviscoelastic simulation of the unconfined compression of articular cartilage: I-simultaneous prediction of reaction force and lateral displacement. Journal of Biomechanical Engineering, 123:191-197, 2001.

[18] S. Federico, A. Grillo, and W. Herzog. A transversely isotropic composite with a statistical distribution of spheroidal inclusions: a geometrical approach to overall properties. Journal of the Mechanics and Physics of Solids, 52(10):2309-2327, 2004.

[19] S. Federico, A. Grillo, G. La Rosa, G. Giaquinta, and W. Herzog. A transversely isotropic, transversely homogeneous microstructural-statistical model of articular cartilage. Journal of Biomechanics, 38(10):2008-2018, 2005. 
[20] L. Han, A.J. Grodzinsky, and C. Ortiz. Nanomechanics of the cartilage extracellular matrix. Annual Review of Materials Research, 41:133-168, 2011.

[21] W.A. Hodge, R.S. Fuan, K.L. Carlson, R.G. Burgess, W.H. Harris, and R.W. Mann. Contact pressures in the human hip joint measured in vivo. Proceedings of the National Academy of Sciences of the United States of America, 83:2879-2883, 1986.

[22] M.H. Holmes. Introduction to Perturbation Methods. Springer, New York, USA, 2013.

[23] G.A. Holzapfel. On large strain viscoelasticity: continuum formulation and finite element applications to elastomeric structures. International Journal for Numerical Methods in Engineering, 39:3903-3926, 1996.

[24] P. Howell, G. Kozyreff, and J. Ockendon. Applied Solid Mechanics. Cambridge University Press, Cambridge, UK, 2009.

[25] J.M. Huyghe and J.D. Janssen. Quadriphasic mechanics of swelling incompressible porous media. International Journal of Engineering Science, 35:793-802, 1997.

[26] J.P. Johnston. The viscosity of normal and pathological human synovial fluids. Biochemical Journal, 59:633-637, 1955.

[27] V. Klika, E.A. Gaffney, Y-C. Chen, and C.P. Brown. An overview of multiphase cartilage mechanical modelling and its role in understanding function and pathology. Journal of the Mechanical Behavior of Biomedical Materials, 62:139-157, 2016.

[28] V. Klika, J.P. Whiteley, C.P. Brown, and E.A. Gaffney. The combined impact of tissue heterogeneity and fixed charge for models of cartilage. Biomechanics and Modeling in Mechanobiology, 2019.

[29] P. Knox, J.R. Levick, and J.N. McDonald. Synovial fluid - its mass, macromolecular content and pressure in major limb joints of the rabbit. Quarterly Journal of Experimental Physiology, 73:33-45, 1988.

[30] W.M. Lai, J.S. Hou, and V.C. Mow. A triphasic theory for the swelling and deformation behaviors of articular cartilage. Journal of Biomechanical Engineering, 113:245-258, 1991.

[31] G.E. Lang, P.S. Stewart, D. Vella, S.L. Waters, and A. Goriely. Is the Donnan effect sufficient to explain swelling in brain tissue slices? Journal of the Royal Society Interface, 11:20140123, 2014.

[32] F.C. Linn and L. Sokoloff. Movement and Composition of Interstitial Fluid of Cartilage. Arthritis and Rheumatism, 8(4):481+, 1965.

[33] C.W. MacMinn, E.R. Dufresne, and J.S. Wettlaufer. Large deformations of a soft porous material. Physical Review Applied, 5:044020, 2016.

[34] V.C. Mow, M.K. Kwan, W.M. Lai, and M.H. Holmes. A finite deformation theory for nonlinearly permeable soft hydrated biological tissues, pages 153-179. Springer-Verlag, New York, 1986.

[35] V.C. Mow, A. Ratcliffe, and A.R. Poole. Cartilage and diarthrodial joints as paradigms for hierarchical materials and structures. Biomaterials, 13:67-97, 1992. 
[36] J.S. Newman and K.E. Thomas-Alyea. Electrochemical Systems. Wiley Interscience, 2004.

[37] L. Ng, A.J. Grodzinsky, P. Patwari, J. Sandy, A. Plaas, and C. Ortiz. Individual cartilage aggrecan macromolecules and their constituent glycosaminoglycans visualized via atomic force microscopy. Journal of Structural Biology, 143:242-257, 2003.

[38] A.N. Norris and M.A. Grinfeld. Nonlinear poroelasticity for a layered medium. The Journal of the Acoustical Society of America, 98:1138-1146, 1995.

[39] R.D. O’Dea, M.R. Nelson, A.J. El Haj, S.L. Waters, and H.M. Byrne. A multiscale analysis of nutrient transport and biological tissue growth in vivo. Mathematical Medicine and Biology, 32:345-366, 2015.

[40] R. Penta, D. Ambrosi, and A. Quarteroni. Multiscale homogenization for fluid and drug transport in vascularized malignant tissues. Mathematical Models and Methods in Applied Sciences, 25:79$108,2015$.

[41] R. Penta, D. Ambrosi, and R.J. Shipley. Effective governing equations for poroelastic growing media. Quarterly Journal of Mechanics and Applied Mathematics, 67:69-91, 2014.

[42] R. Phillips, J. Kondev, J. Theriot, and H.G. Garcia. Physical Biology of the Cell. Garland Science, New York, 2013.

[43] G. Richardson. A multiscale approach to modelling electrochemical processes occurring across the cell membrane with application to transmission of action potentials. Mathematical Medicine and Biology, 26:201-224, 2009.

[44] R.J. Shipley and S.J. Chapman. Multiscale modelling of fluid and drug transport in vascular tumours. Bulletin of Mathematical Biology, 72:1464-1491, 2010.

[45] E.E. van Haaften, K. Ito, and C.C van Donkelaar. The initial repair response of articular cartilage after mechanically induced damage. Journal of Orthopaedic Research, 35(6):1265-1273, 2017.

[46] G.Z. Voyiadjis and A. Samadi-Dooki. Hyperleastic modeling of the human brain tissue: Effects of no-slip boundary condition and compressibility on the uniaxial deformation. Journal of the Mechanical Behavior of Biomedical Materials, 83:63-78, 2018. 